\title{
Collective Particle Flow through Random Media
}

\author{
Joe Watson and Daniel S. Fisher \\ Physics Department \\ Harvard University
}

(Submitted to Phys. Rev. B. on September 20, 1995)

\begin{abstract}
A simple model for the nonlinear collective transport of interacting particles in a random medium with strong disorder is introduced and analyzed. A finite threshold for the driving force divides the behavior into two regimes characterized by the presence or absence of a steady-state particle current. Below this threshold, transient motion is found in response to an increase in the force, while above threshold the flow approaches a steady state with motion only on a network of channels which is sparse near threshold. Some of the critical behavior near threshold is analyzed via mean field theory, and analytic results on the statistics of the moving phase are derived. Many of the results should apply, at least qualitatively, to the motion of magnetic bubble arrays and to the driven motion of vortices in thin film superconductors when the randomness is strong enough to destroy the tendencies to lattice order even on short length scales. Various history dependent phenomena are also discussed.

05.60.+w, 74.60.Ge, 62.20.Fe
\end{abstract}

Typeset using REVTEX 


\section{INTRODUCTION}

The collective transport of classical objects through random media exhibits many interesting features. In the presence of an applied driving force, steady-state motion is usually only possible, in the absence of fluctuations, if the driving force is sufficiently large. The behavior in the vicinity of the threshold force provides a rich collection of different types of non-equilibrium dynamic critical phenomena. These systems can be divided, roughly, into two broad classes, depending on the relative strength of the interactions of the objects among themselves and the interactions with the random medium, the 'pinning'.

If the pinning is, in some sense, weak compared to the interactions among the transported objects then these can form an extended elastic structure. Transport can then be considered as the motion and distortion of this structure. Examples of this elastic class are domain wall motion in magnets, flux lattices, 10 : 11 the interfaces of wetting fluids invading porous media, 12 - 6 contact lines of spreading droplets on rough surfaces, 17,18 surface growth by random deposition, 19 and propagation of burning fronts.20

However if the pinning is strong enough then any elastic structure will break up and the transport will become inhomogeneous with, at least in some regimes, flow occuring along channels determined by the spatial disorder of the random medium. This type of plastic or channel flow may be found for strongly pinned flux lines in (at least) thin film superconductors, 21,22 for magnetic bubble motion in the presence of impurities, 23 for invasion of non-wetting fluids into porous media12.24 (at large enough values of the contact angle),25 29 for fluid flow down a rough or dirty surface,3, 31 for charge transport in small metallic dots, 32 for dielectric breakdown, 33 and for vortex motion in disordered Josephson junction arrays.34

The driven transport of elastic media has been extensively studied in recent years. Much less is known about channel flow, and previous analytic work 30 was restricted to the transport of a continuous fluid and concentrated primarily on the behavior below threshold. Here, we consider the motion of discrete particles pushed by a driving force through a random medium 
in the limit that the interactions among the particles essentially just cause constraints on the local number density.

One of the systems of primary interest is vortex motion in strongly disordered thin film type-II superconductors. Promise of experimental observations of the dynamics of this system was sparked by recent experiments that imaged the vortex lines directly in realtime. 35 With very strong pinning the intervortex lattice correlations will be destroyed and, in a thin film, the vortices will behave roughly as point objects. The driving force is provided by an applied transport current and we will particularly be interested in the behavior just above the critical current for the appearance of steady-state vortex flow. The spatial patterns of the vortex flow, their reproducibility in a given sample, and various kinds of transients will be the focus of our attention.

Although the simple models that we introduce are mostly applicable for very strong pinning, we will argue that on long length scales, the breaking of the lattice which occurs at large scales even for weak pinning, 36 may lead to qualitatively similar behavior - in particular the concentration of the vortex flow near the critical current into relatively narrow, well separated channels.

\section{A. Outline}

The remainder of the Introduction introduces a very simple model and discusses some of the qualitative types of phenomena that it exhibits. Various variants of the model and its behavior above threshold are analyzed in a mean field approximation in Sec. II and in two dimensions in Sec. [II]. In Sec. IV some aspects of the transient behavior below threshold are discussed. Transients and history dependence are considered in Sec. $\nabla$. Applications to

experimental systems and conclusions make up Sec. VI. Some of the technical details of the mean field theory below threshold are relegated to the Appendix. 


\section{B. Models}

We introduce a simple model with several features which can be changed and simplified to allow more information to be obtained. Somewhat different variants are convenient for investigating different aspects of the problem.

The basic model consists of particles on a lattice which move in downhill directions from site to site. The randomness of the medium is represented by a random capacity for each site - the number of particles it can hold without 'overflowing' — and a random local rule for which sites the particle will move to when a given site overflows. Most of the discussion will be about two dimensional systems or mean field approximations for these. We thus, for simplicity, focus on a square lattice. One of the diagonal directions of the lattice is designated as the 'downhill' direction. The applied force acts in this direction which will be referred to as 'vertical'. Directions perpendicular to the vertical will be called 'transverse'. Particles are only allowed to move between nearest neighbor sites along the two possible downhill directions. Therefore each lattice site has two inlets and two outlets as shown in Fig. 11.

If the number of particles on a site exceeds its capacity, then the excess particles will move out. If there is just one excess particle then it will overflow through the primary outlet path of the site which is fixed independently for each site to be to the left or the right. This

is the essential feature of the local randomness in the medium. The case in which more than one excess particle is present will be discussed shortly.

The simplest dynamics are synchronous. Every site on the lattice is simultaneously updated. At any site where the number of particles present exceeds the local capacity, those excess particles are moved along one of the two outlet paths into one of the sites in the next row of the lattice downhill. This synchronous dynamics has the special feature that if all the sites are either full or overflowing, the below capacity particles are inert while the excess particles in one row all move down to the next row together and do not interact or mix with the excess particles in other rows. It would be more realistic to allow somewhat 
asynchronous dynamics. This would result in variations in the vertical speed of the excess particles and hence mixing of the rows. Some features of this will be discussed in Sec. IIIB, but for the most part we will study the synchronous dynamics because of its simplicity.

When particles arrive at a site two things can happen. First, if the site is not full, some or all of the particles can be trapped at that site. Particles that are not trapped will, at the next time step, leave the site through one of the two outlets. If more than one particle leaves, they can either all leave through the primary outlet or the particles can be divided among the two outlets, a split. The way that this choice is decided and the manner in which the particles split is governed by the local splitting rule and the distribution of its parameters.

A simple rule would be to choose the barrier height of each of the two outlets from some distribution. If the number of particles is less than the lower outlet barrier, then no particles leave. If the number of particles at the site exceeds only one of the outlet heights then all of the particles above the lower outlet's height leave by that outlet, i.e., the lower outlet barrier determines the primary outlet. Conversely, if the number of particles exceeds the height of both outlets then the number above the higher outlet could, for example, be evenly divided between both outlets, with an extra one going out the higher outlet if the number that exceed the higher barrier is odd.

Although this splitting rule is suitable for use in simulations, for analytic calculations it is useful to simplify further. This will be done in different ways for various calculations. The important aspects to preserve are that the rule is influenced by the quenched randomness of the outlet connections - particularly by which is the lower outlet - and that a site with more excess particles is more likely to split them among the two outlets

The usual initial condition will be to place particles randomly at each site. Only the number of particles at each site relative to its capacity can play a role. We can thus choose this independently for each site, from a distribution that, in general, will have weight for both positive (excess) and negative (unfilled) values. If we are studying the steady-state behavior above threshold, then it will sometimes be convenient to only allow sites to start at capacity or above. This removes the site-filling transients and simplifies the behavior. 
The basic two dimensional model is defined concretely as follows: The horizontal rows of a square lattice rotated at $45^{\circ}$ are denoted by $y=1,2,3 \ldots$, numbering from the top down, and the sites on row $y$ by $(x, y)$, with $x+y$ an even integer (see Fig. 11). The (integer) number of particles $n(x, y, t)$ on site $(x, y)$ at time $t$ is measured relative to the lower outlet barrier so that $n<0$ implies that the site is unsaturated. The height of the higher outlet barrier relative to the lower barrier is denoted by an integer $B(x, y, t)$.

If $0<n(x, y, t) \leqslant B(x, y, t)$ then at time $t+1, n(x, y, t)$ particles are moved to the site in the next lower row to which the randomly chosen (but fixed) lower (primary) outlet connects, i.e., to one of the sites $(x \pm 1, y+1)$.

If $n(x, y, t)>B(x, y, t)$ then some fraction of the excess particles $\left(n_{L}\right)$ are moved through the primary, lower outlet and the rest, $n-n_{L}$, through the secondary outlet with higher barrier. The rule mentioned above corresponds to

$$
n_{L}=\left\{\begin{array}{cl}
n & \text { if } n \leqslant B \\
B+\frac{n-B}{2} & \text { if } n-B \text { is positive and even } \\
B+\frac{n-B-1}{2} & \text { if } n-B \text { is positive and odd. }
\end{array}\right.
$$

It is best to think of an integer outlet barrier as being the least integer greater than a continuous valued barrier height. Increasing the drive $F$ then corresponds to lowering all of the randomly distributed barrier heights uniformly, resulting in some fraction of the integer barrier heights decreasing by one. This is equivalent to adding a particle to some fraction of the sites, but not quite equivalent to adding particles randomly, since decreasing all barriers together should mean that a new particle will not appear a second time on a site until a new one has appeared on all other sites. Nevertheless for most purposes the differences will be unimportant

A particularly simple version of the model - essentially the simplest possible - is the onedeep model. Here we chose all $B(x, y)$ to be equal to one, so that the randomness is only in the choice of which of the two outlets of each site is the lower, and restrict the $n(x, y, t)$ to be no more than two. Then no more than one particle will be moved through any outlet at a 
given time step, so the $n \leqslant 2$ condition will be preserved. Increasing the drive is equivalent to increasing the number of initial particles. We discuss some other variants in later sections.

For many purposes we will be interested in large systems so that steady states can be established very far from the boundaries, but it is also important to consider the type of boundary conditions appropriate in a finite system. In particular, unless extra particles are added at the top of the system all currents will be transient. Above threshold it is therefore necessary to add particles to the top row in some way. One possibility is to drive the system at constant current. An alternative is to allow all particles moving out from the bottom row to leave the system and to feed particles into the top row at the same rate. In either case, the particles added in the top row could be placed in a regular pattern, a fixed random pattern or in time-varying random positions. Any of these choices might be experimentally relevant.

For computer simulations, periodic boundary conditions in the transverse direction are the obvious choice, so that any particles leaving the left hand side of the system appear immediately at the right hand side. It can also be useful to enforce periodic boundary conditions in the vertical direction giving the system a toroidal topology, with any particle leaving the bottom row being placed in the corresponding column in the top row.

Another choice would be to employ open boundary conditions at top and bottom (no particles enter the top, particles can leave from the bottom) and to add particles to all sites in the system stochastically. This provides a very simple model for rain falling on a dirty windshield 31 As the system becomes longer in the vertical direction the rate of rainfall must be decreased concomitantly in order for a well-behaved large system limit to be reached, we will not study this here.

\section{Qualitative Phenomena}

The behavior of even our very simple model is very rich, exhibiting two distinct 'equilibrium' phases, novel critical behavior, as well as various interesting transient and time 
dependent effects.

Some of the transient and history dependent effects are very subtle. We will thus first describe the behavior below threshold and above threshold in situations where these effects do not play a role.

\section{Below Threshold}

If the driving force is low, there will be few excess particles and they will move downhill readily finding unsaturated sites ('traps') to fall into and stop. As the force is increased further the extra excess particles will again fall, but now have to go further before finding unsaturated sites to fill.

In general this process will be collective and rather complicated: several particles can end up at the same saturated site at the same time raising the level there above the higher outlet barrier and hence splitting the excess particles into two paths. Thus even the route that a given particle will take depends on the other particles.

There is a simple limit, however, where this does not occur. If initially there are no excess particles and then the force is increased very slowly - adiabatically - each excess particle that appears will be able to fall until it finds an unsaturated site at which to stop before the next particle appears. The path of each particle will then entirely be determined by the unique primary (lowest) outlet path that emerges from its original site. Only where on that path it comes to rest will be determined by the positions of other particles. Furthermore, the order in which excess particles appear will not affect the state of the system after they have all come to rest. The behavior in this limit is very similar to that in the continuum 'river' model below threshold studied by Narayan and Fisher (NF). B0

The saturated sites and primary outlet paths that connect them will form tree structures, as shown in Fig. 2, with the primary outlet of the furthest downhill site of the tree going to an unsaturated terminus site. Any excess particle that appears on the tree will fall to this terminus site. If this terminus thereby becomes filled, the tree will connect to another tree 
with a terminus further downhill.

Thus as the drive $F$ increases, some of the trees will become larger and larger (even though most will remain small), forming fractals with typical number of sites scaling with their vertical extent $L$ as $L^{d_{f}}$ with $1<d_{f}<2$ in two dimensions. At a critical drive $F_{a}$, corresponding to a critical distribution of the number of excess particles before they move and the barrier heights, the vertical length of the longest characteristic trees,

$$
\xi_{-} \sim \frac{1}{\left(F_{a}-F\right)^{\nu_{a}}}
$$

will diverge, concomitantly the characteristic time scale for most of the excess particles to come to rest will diverge proportional to $\xi_{-}$, since it takes a time $\xi_{-}$to fall down a tree of length $\xi_{-}$.

The statistics of the saturated trees should be in the same universality class as in the continuum river network case studied by NF. In the Appendix the mean field analysis is explicitly carried out for our discrete particle model and compared with the continuum fluid results.

In two dimensions, NF's numerical results and scaling laws yield

$$
\begin{aligned}
& d_{f}=1.21 \pm 0.02 \\
& \nu_{a}=1.76 \pm 0.02 .
\end{aligned}
$$

Unfortunately in NF's paper, an erroneous argument was given that the width $W$ of a tree of length $L$ would scale as $L^{1 / 2}$. This, by analogy with conventional directed percolation, may well not be correct. 37 More generally we expect

$$
W \sim L^{\alpha_{a}}
$$

The numerics suggest that $\alpha_{a}$ is close to $1 / 2$. Thus the width of the largest characteristic clusters diverges as

$$
W \sim\left(F_{a}-F\right)^{-\alpha_{a} \nu_{a}}
$$


near the threshold. Any error in assuming $\alpha_{a}=1 / 2$ would mean that the error bars quoted in Eq. (3) are too small. The numerics were done in a way that makes the true error bars difficult to reanalyze.

\section{Flowing Phase}

The adiabatic approximation breaks down at threshold. If more particles are added, some of them will start colliding with each other once they have been 'collected' by the large trees. This will result in splits, forming new outlet paths, filling in of extra sites, and, eventually, in the formation of a network of persistent 'channels' that drain the system. The behavior of the transients and the formation of this network we be discussed later, we first consider the behavior after the transients have decayed. An example persistent channel network is shown in Fig 3 .

The simplest way to establish the flowing phase is to insert, at each time step, particles into the top row of the system with a linear density $J$, thereby fixing the input current density. We first consider a time independent source-a fixed set of sites in the top row each receive the same number of particles at each time step. If there were no traps (unsaturated sites) the behavior would be a time independent pattern of channels after the initial input has passed a given row. With traps, all the traps on this equilibrium network will eventually be filled, and afterwards the behavior will again be time independent, depending only on the input sites and current, the outlet paths, and the splitting rules. The initial conditions will affect only the sites that are not on this network. For the time being we ignore these off-network sites. If the current density is small, the network will be sparse with only occasional splits and joins, and most of the outlets will carry a number of excess particles equal to the minimum above which splits occur. The characteristic vertical distance between

channel splits and joins in the flowing phase, $\xi_{+}$, sets the scale above which the current is approximately uniform; it diverges as

$$
\xi_{+} \sim \frac{1}{J^{2}}
$$


for small current density (in two dimensions). This trivial law apparently bears no relationship to the scaling properties below threshold. [Note that $\xi_{+}$must be defined rather carefully; the mean distance between splits is $\mathrm{O}(1 / J)$, see Sec. IIIA.]

For the simple two-dimensional one-deep model, the statistics of the channels far from the top of the system can be worked out exactly and turn out to be surprisingly simple. One interesting feature is that the network turns out to be statistically up-down symmetric, in striking contrast to the strong directionality evident in the saturated trees below threshold.

An important question now arises: does the channel network far from the top of the system depend on which sites the current enters? Of course if all the current is input at one site, the effects of this will persist, we thus restrict consideration to comparing different inputs with the same current density and no long range variations in the density (beyond those expected from, e.g., randomly chosen input sites).

We thus explicitly consider two randomly chosen input configurations in the same system. We show that the difference between the two sets of excess particles after time $t$, i.e., when they are a distance $t$ below the top row, decays as $t^{-1}$ in mean field theory-computed explicitly in Sec. II - and as $t^{-1 / 4}$ in two dimensions, found in Sec. III by analogy with known results for reaction diffusion systems. Thus, far from the top, the channel network for a particular random medium is unique, depending only on the current density.

\section{Transients and History Dependence}

Other than the two simple limits discussed above - adiabatic addition of particles below threshold and current driven behavior above threshold - most of the behavior of the system will depend on both its initial conditions and its history. Here we summarize some of the features.

Below threshold some qualitative features can be readily guessed. If (as in Sec. [C1) the excess particles are added one at a time, the only sites that can possibly become filled by particles not added directly are those which have at least one inlet - a primary inlet — which 
is the primary outlet of a neighbor in the row above. But if many excess particles are added at the same time, enough can arrive at one site to cause overflow to the secondary outlet, thereby moving onto some site which may have no primary inlet. Thus a sudden increase in the force will result in the excess particles spreading out more, filling a different set of sites, and tending to go less far downhill before stopping, than in the adiabatic case.

The filling of sites with no primary inlets (and other extra sites that will also be filled as a consequence of splitting) will make the primary outlet trees have less saturated sites and thus be smaller. This will have the consequence of raising the threshold force, which will, in general, depend on how it is reached.

A specific procedure is to start with a given configuration with no excess particles and suddenly raise the force to $F$, producing many excess particles. When these particles stop moving, there will again be a distribution of saturated trees whose properties can be studied as a function of $F$. This defines a new below threshold critical phenomena problem which contains some of the above threshold physics - splits. If $F$ is greater than some critical $F_{s}$ then the motion of the excess particles will persist indefinitely in an infinite system.

Does this eventual steady-state still have unsaturated sites? The answer to this is by no means obvious, indeed, it depends on defining the limit carefully. In Sec. VB we will argue that indeed for any $F>F_{s}$ reached by suddenly introducing the excess particles at time zero, there will still be unsaturated sites at infinite times and a unique network of channels with time independent flow. The mean current density $J$ will then be a function of $F$ with, one would guess, critical behavior,

$$
J \sim\left(F-F_{s}\right)^{\beta_{s}} .
$$

An alternative way of reaching a steady state current is to adiabatically increase the force, as discussed earlier below threshold. Again an equilibrium channel network will be formed which, we will argue, will be identical to that formed by the sudden network at the same value of the current density (but not the same $F$ ). But a different set of off-channel sites will now be saturated and the current will not behave in the same way as function of $F$, 
perhaps vanishing near the adiabatic threshold $F_{a}$ with a different exponent $\beta_{a}$.

As mentioned in Sec. [C1, much of the behavior on adiabatically increasing $F$ below threshold is closely related to that for the continuum river model discussed in NF. We thus first focus on the behavior above threshold in the presence of a fixed current density.

\section{MEAN FIELD THEORY OF THE FLOWING PHASE}

In many equilibrium and nonequilibrium contexts, various kinds of mean field approximations provide good starting points. In NF it was found that for continuum models below threshold, mean field theory was very instructive but rather subtle. Here we treat the flowing phase of the discrete particle system in a similar mean field manner focusing on the behavior of the channel network. For simplicity we assume that all sites are saturated or have excess particles distributed with mean density $J$. This should apply to the channels after the trapping transients have decayed away.

The crucial feature of the mean field approximation that makes it tractable is to assume that the inputs to any site are uncorrelated. This should be valid, at least formally, if the inter-row connections can be made over arbitrarily long transverse distances instead of between nearest neighbors. We expect also that it will yield correct critical exponents in sufficiently high dimensions.

One row of excess particles will be followed as it moves down the system at constant velocity (set to unity). Because only one row is treated, each lattice site is encountered only once. This means that the fixed, quenched nature of the outlet connections is irrelevant and excess particles within the row join and split stochastically. For convenience, the stochastic dynamics of the joining and splitting is considered in continuous time rather than using discrete time steps. Because of its similarity to an aggregation model 38 this approximation will be referred to as the Smoluchowski approximation. 


\section{A. Smoluchowski Mean Field}

Each site can hold an unrestricted number of particles. It will be convenient to refer to a fixed size of each particle, $b$, to expedite later comparison with flow of a continuous fluid by taking the limit $b \rightarrow 0$.

The state of the row being followed is fully described, because of the assumption of the independence of the inlets, by the function $c(n b, t)$, the concentration of sites that hold $n$ particles at time $t$ within the row.

The concentration of sites that have one or more excess particles (occupied sites) is described by the function

$$
\phi(t) \equiv b \sum_{n=1}^{\infty} c(n b, t)
$$

The factor of $b$ is inserted so that the sum becomes an integral and $\phi$ remains finite in the $b \rightarrow 0$ limit. The current density is given by

$$
J(t) \equiv b \sum_{n=1}^{\infty} n b c(n b, t)
$$

and is trivially equal to the mean number of moving particles per lattice site.

Smoluchowski dynamics are formulated in continuous time. At most times all the particles move down the primary outlet paths. Two types of 'reaction' are possible, particles from two sites can join together and move to a single site (a site to which both inlets are primary) and a single site can split its particles among two adjacent sites, corresponding to overflow through the secondary outlet.

Joining is described by the reaction

$$
[n]+[m] \longrightarrow[n+m]
$$

The notation $[n]$ refers to a site with $n$ particles. This occurs at a rate $\frac{1}{2} \kappa b c(n b) c(m b)$ for each ordered pair $(n, m)$.

Splitting involves reactions of the form 


$$
[n] \longrightarrow[m]+[n-m]
$$

where $m$ is an integer and $0<m<n$. A simple choice is for each of the possible splitting reactions to occur at a rate $\frac{1}{2} \mu b c(n b)$. This choice has the desired feature that a site with a larger number of particles is more likely to split (because of the larger number of accessible values for $m)$. The total splitting rate for sites with $n$ particles will be $\frac{1}{2} \mu(n-1) b c(n b)$.

The presence of empty sites in these reactions is not included directly, this is reasonable when occupied sites are dilute. For regimes where almost all sites are occupied it might be more realistic to consider splitting terms proportional to $c(n) c(0)$ to reflect the fact that an empty site is required for the splitting reaction since $[n] \rightarrow[m]+[n-m]$ is really $[n]+[0] \rightarrow[m]+[n-m]$. We will not analyze this complication here.

The constants $\kappa$ and $\mu$ control the overall time scale and the relative ease of joining and splitting. The factors of $b$ ensure that the total rates are finite in the continuous fluid limit $b \rightarrow 0$. For convenience we set $b=1$ henceforth until we need to consider the limit $b \rightarrow 0$ (Sec. [1B).

The reactions in Eq. (10) and (11) combine to give the evolution equation for $c(n, t)$,

$$
\begin{aligned}
\frac{\partial}{\partial t} c(n, t) & =\frac{\kappa}{2} \sum_{m=1}^{n-1} c(m) c(n-m)-\kappa c(n) \sum_{m=1}^{\infty} c(m) \\
& -\frac{\mu}{2}(n-1) c(n)+\mu \sum_{m=n+1}^{\infty} c(m) .
\end{aligned}
$$

The parameter $\mu$ sets the basic (inverse) time scale while the ration $\mu / \kappa$ sets the overall scale for the concentrations. We rescale the concentrations, $c \rightarrow c \mu / \kappa$, and times, $t \rightarrow t / \mu$ to get rid of $\mu$ and $\kappa$.

In terms of the transformed variable

$$
\gamma(\lambda, t) \equiv \sum_{n=1}^{\infty} e^{-\lambda n} c(n, t),
$$

the evolution equation now becomes

$$
\frac{\partial \gamma}{\partial t}=\frac{1}{2} \gamma^{2}-\phi(t) \gamma+\frac{1}{2} \frac{\partial \gamma}{\partial \lambda}+\frac{1}{2} \gamma+\frac{e^{-\lambda} \phi(t)-\gamma}{1-e^{-\lambda}} .
$$


Given a knowledge of $\gamma(\lambda, t)$, it is easy to obtain the concentration of occupied sites, $\phi(t)=$ $\gamma(0, t)$, and the current density

$$
J(t)=-\left.\frac{\partial \gamma(\lambda, t)}{\partial \lambda}\right|_{\lambda=0},
$$

which is conserved.

A simpler measure of the evolution of the system is provided by the concentration of occupied sites $\phi(t)$. This function is also needed to construct an explicit differential equation for $\gamma(\lambda, t)$. The evolution of $\phi(t)$ is given by

$$
\frac{d \phi}{d t}=-\frac{1}{2} \phi^{2}+\frac{1}{2}(J-\phi),
$$

which is the same as the $\lambda \rightarrow 0$ limit of Eq. (14).

The steady-state value $\phi_{\text {eq }}$ is given by the condition $d \phi / d t=0$ and has positive solution

$$
\phi_{\mathrm{eq}}=\frac{1}{2}(\sqrt{1+4 J}-1) .
$$

Equation (16) is a separable first order equation which has solution

$$
\frac{\phi(t)-\phi_{\mathrm{eq}}}{\phi(0)-\phi_{\mathrm{eq}}}=\frac{2 \phi_{\mathrm{eq}}+1}{\left(\phi(0)+\phi_{\mathrm{eq}}+1\right) e^{\left(\phi_{\mathrm{eq}}+1 / 2\right) t}-\phi(0)+\phi_{\mathrm{eq}}} .
$$

The asymptotic approach of $\phi(t)$ to $\phi_{\mathrm{eq}}$ is given by

$$
\frac{\phi(t)-\phi_{\mathrm{eq}}}{\phi(0)-\phi_{\mathrm{eq}}} \approx \frac{2 \phi_{\mathrm{eq}}+1}{\phi(0)+\phi_{\mathrm{eq}}+1} e^{-t / \tau_{1}},
$$

where the time scale is

$$
\tau_{1}=\frac{1}{\phi_{\mathrm{eq}}+1 / 2}=\frac{2}{\sqrt{1+4 J}} .
$$

Observe that, perhaps surprisingly, this timescale is finite in the limit $J \rightarrow 0$.

The steady state solution for $\gamma$ is found by taking $\partial \gamma / \partial t=0$ in Eq. (14). This gives a Ricatti equation for $\gamma_{\mathrm{eq}}(\lambda)$,

$$
-\frac{1}{2} \frac{d \gamma_{\mathrm{eq}}}{d \lambda}=\frac{1}{2} \gamma_{\mathrm{eq}}^{2}-\left(\phi_{\mathrm{eq}}+\frac{1}{2}+\frac{1}{e^{\lambda}-1}\right) \gamma_{\mathrm{eq}}+\frac{\phi_{\mathrm{eq}}}{e^{\lambda}-1}
$$


The solution must satisfy the boundary condition

$$
\left.\frac{d \gamma_{\mathrm{eq}}}{d \lambda}\right|_{\lambda=0}=-J
$$

and respect the symmetry

$$
\gamma(\lambda+2 \pi i)=\gamma(\lambda)
$$

required by the restriction that $c(n)$ be non-zero only for $n \in\{1,2,3 \ldots\}$.

The only suitable solution is thus

$$
\gamma_{\mathrm{eq}}(\lambda)=\frac{\phi_{\mathrm{eq}}}{\left(\phi_{\mathrm{eq}}+1\right) e^{\lambda}-\phi_{\mathrm{eq}}}
$$

whose transform yields the steady state solution

$$
c_{\mathrm{eq}}(n)=\left(\frac{\phi_{\mathrm{eq}}}{\phi_{\mathrm{eq}}+1}\right)^{n}=\left(\frac{\sqrt{1+4 J}-1}{\sqrt{1+4 J}+1}\right)^{n} .
$$

This can be written

$$
c_{\mathrm{eq}}(n)=e^{-n / \Upsilon}
$$

with characteristic scale for decay of the number of particles at an occupied site

$$
\Upsilon=\frac{1}{\ln \left(1+1 / \phi_{\mathrm{eq}}\right)} .
$$

Although the general dynamics of $\gamma(\lambda, t)$ do not satisfy detailed balance, detailed balance is recovered in the steady state distribution. The rate at which particles move from sites with $n$ particles to sites with $m>n$ particles is

$$
\Gamma_{n \rightarrow m}(t)=\frac{n}{2} c(n, t) c(m-n, t)
$$

The reverse rate of transfer is

$$
\Gamma_{m \rightarrow n}(t)=\frac{n}{2} c(m, t)
$$

These two rates are equal with the equilibrium form of $c_{\mathrm{eq}}(n)$ shown in Eq. (24). 
This suggests that there is substantial simplicity in the steady state. We will see later that, in fact, in a truncated model, all allowed configurations are equally likely.

The discrete nature of the particles is most important in the limit of a small current, $J \rightarrow$ 0 . In this limit the steady-state current distribution is dominated by sites with only a single particle. The concentrations for sites with large numbers of particles become exponentially small, dropping by a factor of approximately $J$ for each additional particle

$$
\frac{c_{\mathrm{eq}}(n+1)}{c_{\mathrm{eq}}(n)}=J+\mathrm{O}\left(J^{2}\right)
$$

and correspondingly

$$
\Upsilon \sim \frac{-1}{\ln J}
$$

As $J \rightarrow 0$, the fraction of particles on sites with only a single particle approaches unity like

$$
\frac{c_{\mathrm{eq}}(1)}{\sum_{n}^{\infty} n c(n)}=1-2 J+\mathrm{O}\left(J^{2}\right) .
$$

It follows that the number of occupied states must be be proportional to the current,

$$
\phi_{\mathrm{eq}} \sim J+\mathrm{O}\left(J^{2}\right) .
$$

The correlation length $\xi_{+}$can be defined as the vertical distance over which the current is correlated. In mean field, this is the same as the typical downhill distance between splits in the channel network, so the rate of splitting is inversely proportional to $\xi_{+}$, just as in NF.

The rate for splitting at any site is given by

$$
\frac{1}{2} \sum_{n=1}^{\infty}(n-1) c(n)=\frac{1}{2}\left(J-\phi_{\text {eq }}\right)=\frac{1}{2} \phi_{\text {eq }}^{2} .
$$

The rate for splitting among occupied sites is therefore $\frac{1}{2} \phi_{\text {eq }}$ and thus, with the particle velocity set to unity, the typical distance a particle travels before reaching a splitting site of the network is

$$
\xi_{+}=\frac{2}{\phi_{\mathrm{eq}}} \sim \frac{1}{J}
$$


in the small current limit.

The relaxation time for approach to the steady state is given by

$$
\tau_{1}=2-4 J+\mathrm{O}\left(J^{2}\right)
$$

This approaches a finite limit as $J \rightarrow 0$. The limiting time-scale is set by the splitting rate $[1 / \mu$ in Eq. (12)]. No joining is necessary for equilibration in this mean field approximation. The finite relaxation time is obtained because, although $d c(n, t) / d t$ vanishes as $J^{n}$, the equilibrium concentration $c_{\mathrm{eq}}(n)$ is also of order $J^{n}$

The finite relaxation time is only for the equilibration of the distribution of excess particle numbers. The time for spatial correlations of the equilibrium network to develop does diverge as $J \rightarrow 0$, but this is not seen by studying only a single row. We will return to this in Sec. [ID.

\section{B. Comparison with Continuous Fluid}

A mean field model with a continuous fluid, like those studied by NF, can be recovered by reinstating the particle size $b$ and then taking the limit $b \rightarrow 0$. The results obtained for a small current are then different from the discrete case, that is the limits $J \rightarrow 0$ and $b \rightarrow 0$ do not commute.

In the continuum limit the concentration $c(x)$ is a function of a continuous variable and the sum $\phi$ is replaced by the integral,

$$
\phi(t)=\int_{0}^{\infty} d x c(x, t)=\lim _{b \rightarrow 0} \sum_{n=1}^{\infty} b c(n b, t)
$$

and the current is defined by

$$
J(t)=\int_{0}^{\infty} d x x c(x, t)=\lim _{b \rightarrow 0} \sum_{n=1}^{\infty} n b^{2} c(n b, t) .
$$

The steady state value of $\phi$ is

$$
\phi_{\mathrm{eq}}=\lim _{b \rightarrow 0} \frac{b}{2}\left(\sqrt{1+\frac{4 J}{b^{2}}}-1\right)=\sqrt{J}
$$


This value of $\phi_{\text {eq }}$ shows that the fraction of sites that are in channels vanishes much more quickly for small current in the continuous case $\left(\phi \sim J^{1 / 2}\right)$ than in the discrete case $(\phi \sim J)$. This is because in the discrete case the mean number of particles at an occupied site $(J / \phi)$ cannot be less than one, whereas in the continuous case the mean amount of fluid at each site approaches zero as $J \rightarrow 0$.

The steady-state site concentration distribution can be obtained from the transform

$$
\gamma_{\mathrm{eq}}(\lambda)=\lim _{b \rightarrow 0} \frac{b \phi_{\mathrm{eq}}}{\left(\phi_{\mathrm{eq}}+b\right) e^{\lambda b}-\phi_{\mathrm{eq}}}=\frac{1}{\lambda+1 / \phi_{\mathrm{eq}}}
$$

yielding

$$
c_{\mathrm{eq}}(x)=\exp (-x / \sqrt{J}),
$$

so that the typical channel site has $\sim \sqrt{J}$ excess fluid.

The vertical correlation length can also be found in the continuum limit:

$$
\xi_{+}=\frac{2}{\phi_{\mathrm{eq}}}=\frac{2}{\sqrt{J}} ;
$$

it diverges more slowly than Eq. (34), where $\xi_{+} \sim 1 / J$. Finally the time scale for equilibration of the single site properties, such as $\phi$, in a continuum fluid is

$$
\tau_{1}=\frac{1}{\phi_{\mathrm{eq}}},
$$

which diverges as $J \rightarrow 0$ in contrast to the discrete case.

In the continuous fluid, $\xi_{+}$and $\tau_{1}$ both diverge as $J^{-1 / 2}$. This divergence should be expected: as $J \rightarrow 0$ the rivers get shallower and split less often, increasing the size of the flow pattern $\left(\xi_{+}\right)$and requiring longer to equilibrate $\left(\tau_{1}\right)$. In contrast, in the discrete case $\xi_{+}$ diverges simply (as $J^{-1}$ ) as the channel sites get further apart, but the time scale, $\tau_{1}$, found above does not diverge. If we consider, for example, an initial condition where there are single isolated particles on small fraction $J$ of the sites, then the steady state number of sites with each occupation has almost been reached, so $\tau_{1}$ is small and finite, and the vertical length scale for the flow pattern is $J^{-1}$, the joining rate. 
Note that the behavior of the continuous fluid in the limit of small current depends (in contrast to the discrete case) on the splitting rule. The splitting rule used here and in NF are thus appropriate for a distribution of the outlet barrier differences $B$ which is constant near $B=0$. A different form of the distribution for small $B$ will result in different exponents characterizing the small current behavior since the splitting rate of shallow rivers will be a different function of their depth. This is somewhat analogous to the dependence of certain exponents in conventional continuum percolation on local properties of the medium.39

\section{Restricted Depth Models}

In the Smoluchowski mean field calculation we found that the steady-state concentration of sites containing $n$ particles scales as [Eq. (29)]

$$
c(n) \sim J^{n},
$$

when the mean current of particles $J$ is small. This suggests that, at small $J$, a reasonable approximation will be to limit the possible number of particles at each site to

$$
0 \leqslant n \leqslant N
$$

for some constant value $\mathrm{N}$.

This restriction makes theoretical treatment of the model much simpler.

The 'depth' restriction can conveniently be enforced for even values of $N$ by allowing no more than $N / 2$ particles to travel down any outlet in one time-step. We will consider two versions, a 'one-deep' model, which allows only one particle to travel through any outlet, and a 'two-deep' model, which allows two.

For the one-deep model, mentioned in the Introduction, each site can hold at most two particles (i.e., $N=2$ ). This is preserved by using synchronous dynamics and allowing no more than one particle to pass along any outlet. If a site holds one particle, then the particle moves onto the next row through the primary outlet. If the site has two particles, one particle moves through each of the two outlets. 
The rules are similar for the two-deep model. A site can have up to four particles $(N=4)$ and each outlet can pass a maximum of two particles. A site with one particle passes that particle to the primary outlet. A site with four particles passes two particles to each outlet. A site with three particles passes two to the primary outlet and one to the secondary outlet. A site with two particles can either pass both particles to the primary outlet or it can pass one particle to each of the two outlets. This choice is fixed independently for each site with probability $r$ of the site sending both particles through the same outlet. The choice $r=1 / 3$ makes the mean-field calculation simpler and will be treated separately.

Both of these models have a particle-hole symmetry. A near-maximal current behaves the same way as a very small current. The truncation that creates these models is of course only reasonable in the latter case.

As for the Smoluchowski mean field, we choose a particular row of particles and follow that row as it moves down through the system. The probability that a site in that row holds $n$ excess particles at time $t$, is given by $p(n, t)$. With synchronous, discrete time dynamics the probabilities at time $t+1$ depend only on those at time $t$, so we will construct a set of recursion relations giving $p(n, t+1)$ in terms of $p(n, t)$. For brevity we will write these as $p_{n}^{\prime}$ and $p_{n}$ respectively.

To calculate the values $p_{n}^{\prime}$ for a given site, $\mathrm{X}$, we need to look at the two sites (say $\mathrm{V}$ and $\mathrm{W}$ ) which provide inputs to $\mathrm{X}$. The inlets VX and WX are each equally likely to be primary or secondary outlets, so each combination of input types occurs with probability one quarter. The simplest way to calculate $p_{n}^{\prime}$ for $\mathrm{X}$ is to consider every possible combination of inlet types and of occupations for $\mathrm{V}$ and $\mathrm{W}$. For each combination the new occupation of $\mathrm{X}$ is given by the splitting rules discussed above.

\section{One-deep}

For the one-deep case, we obtain

$$
p_{0}^{\prime}=p_{0}^{2}+p_{0} p_{1}+\frac{1}{4} p_{1}^{2}
$$




$$
\begin{aligned}
& p_{1}^{\prime}=p_{0} p_{1}+p_{1} p_{2}+2 p_{0} p_{2}+\frac{1}{2} p_{1}^{2} \\
& p_{2}^{\prime}=p_{2}^{2}+p_{1} p_{2}+\frac{1}{4} p_{1}^{2} .
\end{aligned}
$$

The analysis can be simplified by using the probabilities $q_{n}$ of $n(=0$ or 1$)$ particles passing through an arbitrary primary or secondary inlet to site $\mathrm{X}$ in one time step:

$$
\begin{aligned}
& p_{0}=q_{0}^{2} \\
& p_{1}=2 q_{0} q_{1} \\
& p_{2}=q_{1}^{2},
\end{aligned}
$$

and, for an outlet from site $\mathrm{X}$, which can be either the primary or secondary outlet (each with probability $1 / 2$ ), we obtain the simple recursion relations

$$
\begin{aligned}
& q_{0}^{\prime}=q_{0}^{2}+q_{0} q_{1} \\
& q_{1}^{\prime}=q_{1}^{2}+q_{0} q_{1},
\end{aligned}
$$

where $q_{n}^{\prime}$ refer to the outlets of a site and $q_{n}$ refer to its inlets. The mean field approximation makes the two inlets independent. The two outlets from a site are correlated but only one outlet from each site need be treated here as the two outlets will go to different ('far away') sites.

For the one-deep case there are two variables and two conserved quantities: the total probability, $q_{0}+q_{1}=1$, and the mean current per site, $J=2 q_{1}$. This means that there are no dynamics in mean field and, trivially,

$$
\begin{aligned}
q_{0} & =\frac{1}{2}(2-J) \\
q_{1} & =\frac{1}{2} J .
\end{aligned}
$$

\section{Two-deep}

The recursion relation for the two-deep model can be derived in the same way as the one-deep model, again working in outlet variables, 


$$
\begin{aligned}
& q_{0}^{\prime}=q_{0}^{2}+q_{0} q_{1}+\frac{1}{2} q_{1}^{2} r+q_{0} q_{2} r \\
& q_{1}^{\prime}=q_{0} q_{1}+q_{1}^{2}(1-r)+2 q_{0} q_{2}(1-r)+q_{1} q_{2} \\
& q_{2}^{\prime}=q_{2}^{2}+q_{1} q_{2}+\frac{1}{2} q_{1}^{2} r+q_{0} q_{2} r .
\end{aligned}
$$

These can be reduced to the single relation

$$
q_{1}^{\prime}=\frac{1}{8} J(4-J)(1-r)+\frac{1}{2} q_{1}^{2}(1-3 r)+q_{1} r
$$

by applying the two conservation laws,

$$
q_{0}+q_{1}+q_{2}=1
$$

and

$$
J=2 q_{1}+4 q_{2}
$$

The two-deep model has one free parameter, the fraction $(r)$ of sites from which, when the site is doubly occupied, both particles will take the same outlet.

The cases $r=0$ and $r=1$ are special. For $r=0$ the depth-two outlets eventually disappear and the equilibrium is the same as the one-deep model. For $r=1$ all sites eventually have an an even number of particles and the model becomes a doubled version of the one-deep model. The case $r=1 / 3$ removes the quadratic term from the $q_{1}$-recursion relation, simplifying the analysis.

For general values of $r$, the recursion relation [Eq. (50)] is quadratic and converges to the fixed point

$$
q_{1}^{\mathrm{eq}}=\frac{1-r-\sqrt{(1-r)[(1-r)+J(1-J / 4)(3 r-1)]}}{1-3 r} .
$$

This steady-state distribution has the small current limit

$$
\begin{aligned}
q_{0}^{\mathrm{eq}} & =1-\frac{1}{2} J+\frac{1}{8} \frac{r}{1-r} J^{2}+\mathrm{O}\left(J^{3}\right) \\
q_{1}^{\mathrm{eq}} & =\frac{1}{2} J+\frac{1}{4} \frac{r}{1-r} J^{2}+\mathrm{O}\left(J^{3}\right) \\
q_{2}^{\mathrm{eq}} & =\frac{1}{8} \frac{r}{1-r} J^{2}+\mathrm{O}\left(J^{3}\right) .
\end{aligned}
$$


Convergence towards this equilibrium is exponential with time-constant

$$
\tau_{1}=\frac{-1}{\ln (1-\sqrt{(1-r)[(1-r)+J(1-J / 4)(3 r-1)]})}
$$

which does not diverge in the physical regime $(0 \leqslant r \leqslant 1$ and $0 \leqslant J \leqslant 4)$ except at $r=1$ and at $r=0, J=2$.

For the case $r=1 / 3$ the recursion relation has the simple linear form

$$
q_{1}^{\prime}-q_{1}=\frac{1}{12} J(4-J)-\frac{2}{3} q
$$

with a single fixed point at

$$
\left(\begin{array}{c}
q_{0}^{\mathrm{eq}} \\
q_{1}^{\mathrm{eq}} \\
q_{2}^{\mathrm{eq}}
\end{array}\right)=\frac{1}{16}\left(\begin{array}{c}
(4-J)^{2} \\
2 J(4-J) \\
J^{2}
\end{array}\right)
$$

and convergence is exactly exponential,

$$
q_{1}^{\prime}-q_{1}^{\mathrm{eq}}=\frac{1}{3}\left(q_{1}-q_{1}^{\mathrm{eq}}\right)
$$

with time scale $1 / \ln 3$. The independence of $J$ is special to this value $(r=1 / 3)$ but the finite convergence rate is more generic.

\section{Comparison with Smoluchowski}

We see that the finite time scale for convergence of the single site distribution exists both for these truncated models and the Smoluchowski model in the mean field limit. Other aspects of the truncated models can be compared with the Smoluchowski mean field. They should be similar for small currents.

The basic features are easily checked for small $J$. The $c_{\text {eq }}(n) \sim J^{n}$ property that motivated the truncation is preserved for both one-deep and two-deep models. In the truncated models this corresponds to the site variables relation $p_{n}^{\text {eq }} \sim J^{n}$ which follows from the fact that the outlet variables obey $q_{n}^{\mathrm{eq}} \sim J^{n}$. Concomitantly, as $J \rightarrow 0$ the fraction of sites that 
have exactly one particle approaches unity linearly with $J$, but with a model dependent coefficient.

The correlation length is given by the scale of splitting in the river network and is inversely proportional to the rate of splitting in the steady state. For the one-deep model all the sites that have two particles split so that

$$
\frac{1}{\xi_{+}} \propto \frac{p_{2}}{1-p_{0}}=\frac{q_{1}^{2}}{1-q_{0}^{2}} \sim J
$$

as $J \rightarrow 0$ just as in the Smoluchowski case [Eq. (34)]. In the two-deep case the splitting rate is proportional to $(1-r) p_{2}+p_{3}+p_{4}$ and is also $\mathrm{O}(J)$ for small $J$.

\section{Convergence to Channel Network}

In this subsection we study the development, in mean field theory, of the channel network. To do this we consider two rows of particles which start with different initial configurations but move in the same realization of the random medium, starting at the same row. This allows us to address some interesting questions. Does the flow from different initial conditions approach a fixed network? If the system is driven by a current at a top boundary how far down does the influence of the distribution of the driving current extend?

We will examine the one-deep and two-deep models first.

\section{One-deep}

In the mean field approximation the state of the two copies of the system is represented by the joint probability $p_{m n}$ that a site in the first copy holds $m$ particles and a site in the second copy holds $n$ particles. For the one-deep system this requires a three-by-three matrix of probabilities

$$
\left(\begin{array}{lll}
p_{00} & p_{01} & p_{02} \\
p_{10} & p_{11} & p_{12} \\
p_{20} & p_{21} & p_{22}
\end{array}\right)
$$


Again, it is simpler to use the outlet representation

$$
\left(\begin{array}{ll}
q_{00} & q_{01} \\
q_{10} & q_{11}
\end{array}\right)
$$

whose state matrix has four parameters, but only three are independent because the total probability must be unity. The current density $J$ which is the same for both copies fixes two combinations of the $q_{m n}$ and thus given one of the entries in the state matrix (say $q_{00}$ ) we know all of the others.

The recursion relations for this system can be written down in just the same way as for the single system. Now the iteration is over the number of particles entering along the inlets in each of the two systems and over whether the outlet (in both systems together) is the primary or secondary:

$$
\left(\begin{array}{cc}
q_{00}^{\prime} & q_{01}^{\prime} \\
q_{10}^{\prime} & q_{11}^{\prime}
\end{array}\right)=\left(\begin{array}{cc}
q_{00}+q_{01} q_{10} & q_{01}-q_{01} q_{10} \\
q_{10}-q_{10} q_{01} & q_{11}+q_{11} q_{00}
\end{array}\right) .
$$

Using the constraints this reduces simply to

$$
q_{00}^{\prime}-q_{00}=\left(1-\frac{1}{2} J-q_{00}\right)^{2},
$$

which yields the simple diagonal steady state matrix

$$
\left(\begin{array}{rr}
q_{00}^{\mathrm{eq}} & q_{01}^{\mathrm{eq}} \\
q_{10}^{\mathrm{eq}} & q_{11}^{\mathrm{eq}}
\end{array}\right)=\left(\begin{array}{cc}
1-J / 2 & 0 \\
0 & J / 2
\end{array}\right),
$$

corresponding to each of the two systems being locked in the same state. Thus, indeed we see that the two initial conditions converge to the same steady state channel network.

Because the recursion relation [Eq. (63)] is a perfect square, the approach to the steady state is only quadratic. If $q_{00}=q_{00}^{\mathrm{eq}}-\delta$ then the change $q_{00}^{\prime}-q_{00}$ is only $\delta^{2}$ which means that the difference between $q_{00}$ and $q_{00}^{\text {eq }}$ decreases like $t^{-1}$. This is a much slower equilibration than for the single site distribution in mean field where the approach was exponential 


\section{Two-deep Model}

To describe two copies in the two-deep model using the outlet representation requires a three-by-three matrix

$$
\left(\begin{array}{lll}
q_{00} & q_{01} & q_{02} \\
q_{10} & q_{11} & q_{12} \\
q_{20} & q_{21} & q_{22}
\end{array}\right)
$$

The recursion relations for the matrix can again be constructed by enumerating all possible inputs to both systems. The fate of doubly occupied sites is, of course, chosen once for each lattice site, with the probability parameterized by $r$, and thus has the same result in each of the two copies.

Because, in mean field, each single system equilibrates exponentially, it is simplest to consider the subspace where each single system is equilibrated (this happened trivially for the one-deep model by specifying $J$ ). This requirement fixes the sum of each row and column of the state matrix so we have only four remaining degrees of freedom, say $\left(q_{00}, q_{01}, q_{10}, q_{11}\right)$. The recursion relation is then between two 4-vectors.

$$
\left(\begin{array}{l}
q_{00} \\
q_{01} \\
q_{10} \\
q_{11}
\end{array}\right) \longrightarrow\left(\begin{array}{c}
q_{00}^{\prime} \\
q_{01}^{\prime} \\
q_{10}^{\prime} \\
q_{11}^{\prime}
\end{array}\right)
$$

The new values of each coefficient are quadratic functions of the four previous coefficients and quartic functions of $J$.

The state where both copies are in the same state is a fixed point of this relation. To show that this is a stable fixed point requires more work. We first consider linear stability analysis with a perturbation around this fixed point 


$$
\left(\begin{array}{c}
q_{00} \\
q_{01} \\
q_{10} \\
q_{11}
\end{array}\right)=\left(\begin{array}{c}
q_{0}^{\mathrm{eq}} \\
0 \\
0 \\
q_{1}^{\mathrm{eq}}
\end{array}\right)+\left(\begin{array}{c}
x_{1} \\
x_{2} \\
x_{3} \\
x_{4}
\end{array}\right)
$$

Because the matrix values are probabilities this means, for this fixed point, only perturbations with $x_{2} \geqslant 0$ and $x_{3} \geqslant 0$ are physical.

We focus on the simple $r=1 / 3$ case. To leading order in the $x_{i}$ variables, the recursion relation for $\vec{x}=\left(x_{1}, x_{2}, x_{3}, x_{4}\right)$ is

$$
\vec{x}^{\prime}=M \vec{x}
$$

where $24 M$ is the matrix

$$
\left(\begin{array}{cccc}
8(5-J) & 4(4-J) & 4(4-J) & 4(4-J) \\
(J-4)(J+8) & J^{2}-8 & 4(J-4) & 4(J-4) \\
(J-4)(J+8) & -4(4-J) & J^{2}-8 & 4(J-4) \\
32+8 J-2 J^{2} & 16+4 J-J^{2} & 16+4 J-J^{2} & 1
\end{array}\right)
$$

The matrix $M$ is not symmetric. It has the eigenvalues $1,1 / 3$ and $\left(J^{2}-4 J+8\right) / 24$, the last being doubly degenerate. The four right eigenvectors are not orthogonal but they span the space and any perturbation can be written as a linear combination of them. We write $\vec{x}$ as a component parallel to the first (right-) eigenvector and a component perpendicular to its corresponding left-eigenvector (i.e., as a linear combination of the other three righteigenvectors)

$$
\vec{x}=a_{1} \vec{e}_{1}+a_{\perp} \vec{e}_{\perp}
$$

Except for the first eigenvalue, the others are never greater than $1 / 3$. This means, that if this perturbation is subjected to the iteration then the perpendicular component will go to zero exponentially on a time scale of $1 / \ln 3$ or less. The long time behavior is therefore controlled by the eigenvector corresponding to the unit eigenvalue. To linear order the 
unit eigenvalue indicates that the perturbation is marginal. Stability can be determined by projecting the full recursion relation onto the direction of the first (right-) eigenvector using the corresponding left-eigenvector. This yields

$$
a_{1}^{\prime}=a_{1}-a_{1}^{2}
$$

This implies that $a_{1}$ decays to zero as $t^{-1}$ whenever $a_{1}$ is positive. Formally the fixed point is unstable to negative $a_{1}$, however this is unphysical because the eigenvector,

$$
\vec{e}_{1}=\left(\begin{array}{c}
-1+J / 4 \\
1-J / 4 \\
1-J / 4 \\
-1
\end{array}\right)
$$

then produces negative entries in the probability matrix. Thus the fixed point with the two copies locked together is marginally stable.

For general values of $r$ the behavior is very similar to that for the case $r=1 / 3$ with the same recursion relation for $a_{1}$. The system will again approach the state where both copies are in the same state, but will do so only as $t^{-1}$.

\section{General Mean field}

The mean field treatment of the two simple models showed that the flow pattern will approach a fixed network but only algebraically with the fraction of sites for which the initial conditions alter the network decaying at long times as $t^{-1}$. A general argument, based on analogy with a reaction diffusion system, shows that in mean field theory this result is general.

The decay can be understood by keeping track of inlets in which the occupation in the two copies differ. For the one-deep model we label each inlet with an extra particle in the first copy 'A' and each inlet with higher occupation in the second copy 'B'. Inlets which have the same occupation in each copy are not labeled. As the row is followed down the 
system, the A's and B's move independently (except that no more than one particle may occupy a site) until an A meets a B. The corresponding particles from then on follow the same path and thus the $\mathrm{A}$ and $\mathrm{B}$ disappear. This therefore corresponds to an $A+B \rightarrow \emptyset$ reaction diffusion system. 40

In the continuous time approximation (like the Smoluchowski model) the $A+B \rightarrow \emptyset$ mean field equations are (in dimensionless form),

$$
\frac{d \rho_{A}}{d t}=\frac{d \rho_{B}}{d t}=-\rho_{A} \rho_{B}
$$

with $\rho_{A, B}$ the concentrations of A's and B's. With the same current density in each copy we begin with equal concentrations of $\mathrm{A}$ and $\mathrm{B}$, and the solution is

$$
\rho_{A}=\rho_{B}=\frac{1}{t}
$$

For the two-deep and more general models, the analysis is rather more complicated since there are a variety of ways in which the two copies can differ. These can combine in different ways as well as some combinations annihilating. In the continuous time approximationgenerally good at long times - there will be a coupled series of quadratic recursion relations for the densities, $\left\{\rho_{i}\right\}$, of sites in which the two copies differ in various ways. These equations will generally have a solution with all the $\rho_{i}$ decaying to zero proportional to $t^{-1}$. As for the two-deep analysis at long times we expect the general initial conditions to decay exponentially to this asymptotic form. Thus the $t^{-1}$ behavior for convergence of two initially randomly different copies will be generic in mean field.

\section{FLOWING PHASE IN TWO DIMENSIONS}

In this section properties of the current distribution in the flowing phase are analyzed for the two dimensional systems of primary interest. Some of the features will be qualitatively similar to those found in mean field theory in the previous section, but of course non-trivial correlations will exist that are absent in mean field theory. We will focus on the one-deep 
model for which a number of exact results can be obtained. In Sec. IIIB the extent to which the results apply more generally will be discussed.

\section{A. One-Deep Model}

The one-deep model defined in the Introduction is the simplest model with the essential features. Each outlet carries either zero or one particles at each time step so any site can contain at most two particles above its capacity.

As in the previous section, we will focus on the behavior after all traps that might be filled have been filled. As discussed in the Introduction this can most easily be achieved by adding particles at a fixed rate in the top row. Thus all accessible sites can be considered to be at or above their capacities.

\section{Single row statistics}

In mean field theory, the only quantity of interest for the evolution of a single row moving down the system is the single outlet occupation distribution which is trivially determined by the current density $J$. In two dimensions any translational invariant distribution for the outlet occupation in a row will again have the same single outlet distribution. Surprisingly, the steady state row distribution turns out to have the same simple product form as in mean field so that the steady state occupation of each outlet in a row is independent. This can be seen to be a steady state by considering the two inlets and two outlets of a given site.

If the two inlets are independently occupied with probability $J / 2$, then at the next time step, the probability of either both or neither of the outlets being occupied are trivially $J^{2} / 4$ and $(1-J / 2)^{2}$ respectively. Although the behavior if exactly one of the inlets is occupied is determined by which of the outlets is the primary outlet, the fact that this is independent from site to site and that each site is only encountered once as a row of particles moves down the system immediately means that outlet occupations 10 and 01 are equally likely with probability $(1-J / 2) J / 2$ independently for each pair. Thus, the outlets are independently 
occupied if the inlets are, proving that the independent occupation distribution is a steady state. We expect that this will be the unique steady state; this should be provable along the lines of the arguments developed below.

\section{Uniqueness of the channel network}

Before considering the nontrivial correlations that exist between rows in the steady state, we first show that, as in mean field, the actual network of channels-i.e., which outlets are occupied - does not depend on the initial conditions. We use arguments analogous to those for the mean field case, considering two copies of the system-specifically following a given

row — with different random initial conditions. The same strategy can be followed as for the mean field treatment of Sec. [ID.

Consider a single inlet on the row to be followed. It contains either zero or one particles in each copy. We can represent these possible states as 00, 01, 10 and 11. Each outlet, the inlets to the next row down at the next time step, also contain zero or one particles in each copy; these are represented similarly.

If one particle enters the first copy of the site through, say, the left inlet and no particles enter the second copy, then the site will output one particle in the first copy and no particles in the second copy. Using our notation for the four sites this can be written

$$
10+00 \longrightarrow 10+00
$$

where the output 10 will be, with equal probability, on the left or right outlet.

If a single particle entered from the same side (e.g., the left) in both copies then one particle would leave in both copies through the same outlet. This can be written

$$
11+00 \longrightarrow 11+00 .
$$

This evolution rule

$$
x+y \longrightarrow x+y
$$


holds for all but one case. If a single particle enters both copies of the site, but the particle enters from different inlets in the two copies, the particle will leave both copies of the site via the same (primary) outlet. This can be written

$$
10+01 \longrightarrow 11+00
$$

The notation can thus be simplified by considering only the 10 and 01 outlets, which can conveniently be called A-states and B-states. Our single non-trivial reaction is then

$$
A+B \longrightarrow \emptyset
$$

as in mean field, where $\emptyset$ represents any combination of 00 and 11, i.e., sites where both copies have the same occupation. Apart from this single reaction, the A and B sites perform independent unbiased random walks along their row with the 'excluded-volume' restriction that no more than one state can be assigned to each outlet. This model is therefore an example of the extensively studied $A+B \rightarrow \emptyset$ reaction diffusion system. 40 , 1 ,

The basic picture which should apply in low dimensions is as follows. In a time $t$, the $\mathrm{A}$ and $\mathrm{B}$ particles will perform random walks in the transverse direction, annihilating if they encounter each other. Since in time $t$, two random walks that start a distance less than $\mathrm{O}\left(t^{1 / 2}\right)$ apart are very likely to intersect, for long times the minority species in regions of width less than $\mathrm{O}\left(t^{1 / 2}\right)$ are likely to be completely annihilated in time $t$ by the majority species in that region. Thus after time $t$, the system will be, roughly, divided into regions of width $\sim t^{1 / 2}$, each of which contains mostly $\mathrm{A}$ or mostly $\mathrm{B}$. The number of A's in an A rich region will be of order the typical excess of A over B in that region initially, which, for initial conditions without long range spatial correlations, will be of order $\left(t^{1 / 2} J\right)^{1 / 2}$ for small $J$. Thus after long time $t$, the density of A's (or B's) remaining will be of order

$$
\rho_{A} \sim \frac{J^{1 / 2}}{t^{1 / 4}}
$$

Note that this will only be valid on times longer than then basic collision time $\left(\sim J^{-2}\right.$ for small $J$ ) between particles, with $\rho_{A} \sim J$ for shorter times. 
Thus we see that the convergence to a unique steady state is a slow power law-slower than in mean field — with the number of 'defects' decaying as $t^{1 / 4}$. Similar arguments should obtain, as long as the number of transverse dimensions, $d-1$, is less than 4 , yielding a defect density decaying as $t^{-(d-1) / 4}$ for $d<5$, the mean field result $t^{-1}$ obtaining for $d>5$, since in high dimensions random walkers are essentially as likely to annihilate with walkers that started far away as with those that started nearby. For the reaction diffusion system, these results have been proven. 42,4

\section{Inter-row correlations}

We have shown that the channel network should be unique far from the top of the system. The correlations within one row for the one-deep model are trivial-just exclusion to no more than one particle on the inlets. But there will be correlations between one row and another or between different times. Since in the steady-state each row of particles will follow the same pattern down the system, the correlations in occupation probabilities will be time independent and those between different rows can be obtained by following the evolution of a single row of particles down the system. We will focus on the correlation function $g(x, y)$, defined as the probability that an outlet at position $\left(x^{\prime}, y^{\prime}\right)$ and one at $\left(x^{\prime}+x, y^{\prime}+y\right)$ are both occupied. Translational invariance of the steady state implies that this is a function of $x$ and $y$ only.

In order to follow the correlations of a row of particles down the system we use a trick which takes advantage of the fact that for this row the direction of the primary outlet at each site is only probed once. This allows a simple transformation to be made.

At each site we have two equally likely possibilities, that the left outlet is primary or that the right outlet is primary. If one particle enters the site then it leaves via the primary outlet which is equally likely to be to the left or right. If zero or two particles enter the site then the properties of the site are irrelevant and the same number of particles pass through both outlets. The probabilities of the various outcomes are exactly preserved if instead of 
choosing between primary-left and primary-right states we choose between 'crossed' and 'uncrossed' configurations. Any particle now follows the continuous route ahead of it. A single particle entering (from either side) is still equally likely to leave to the left or the right. If zero or two particles enter the situation is also unchanged. If two particles enter, then we have, essentially, attached labels to them by distinguishing between the crossed and uncrossed configurations; these labels are useful in computing correlations but, of course, they can not appear in physical quantities.

This picture of particles following predetermined crossing routes is illustrated in Fig. 1 . It shows that the probability that an outlet passes a particle in one row is just the probability that the outlet on any specific earlier row on the same route passed a particle. If the initial condition is that each outlet is independently occupied with probability $J / 2$, as it is in steady state, then this condition is trivially preserved for all rows.

Note that this picture is limited to following down a single row of particles. This can be seen by considering a site in which only one particle enters, but from the left in one copy and the right in another. Since these particles should both go out the same primary outlet, the choice of crossed or uncrossed configurations would need to be made differently for the two copies.

We have mapped the single row evolution to a collection of non-interacting particles. The correlation function is thus very simple in terms of the outlet (or inlet) occupation numbers. The correlation function $g(x, y)$ is determined by whether the two outlets lie on the same route. If they are on different routes then each has an independent probability $J / 2$ of holding a particle, so the probability that both have a particle is $J^{2} / 4$. However if the outlets lie on the same route, they will either both be occupied with probability $\mathrm{J} / 2$ or both be unoccupied. Thus

$$
g(x, y)=\frac{J}{2} f(x, y)+\frac{J^{2}}{4}[1-f(x, y)]
$$

where $f(x, y)$ is the probability that the two outlets lie on the same route. The truncated correlation function is then simply 


$$
g(x, y)-\frac{J^{2}}{4}=\frac{J}{2}\left(1-\frac{J}{2}\right) f(x, y),
$$

the particle-hole symmetry (under $J \rightarrow 2-J$ ) being manifest. The function $f(x, y)$ is just the diffusion kernel on the outlet lattice, and hence for large $y$, scales as

$$
f(x, y) \approx \sqrt{\frac{2}{\pi y}} \exp \left(-x^{2} / 2 y\right)
$$

The simplicity of this correlation function hides some complexity as this treatment considers only one row of the system. The development of correlations between different rows is not obtainable by this trick.

Other information on the channel network is readily available, however. For example, one could ask about the typical vertical distance between splits and joins as a way to define a correlation length. Specifically if there is a split (i.e., a doubly occupied site) at some point $\vec{s}$ in the network then one can consider following the primary path from that point down and asking for the probability of another part of the network joining it as a function of vertical distance. This will have two contributions. For short distances, the dominant processes will be further intersections with the other route that bifurcated at the initial split. The probability of this decays with vertical distance as $y^{-1 / 2}$, just the probability of return to the starting point of a random walk in the transverse direction representing the difference in position between the two routes. But in addition there is the chance that another occupied route will intersect the first. Since the positions of the other particles in the same row as $\vec{s}$ is random, for small $J$ this extra joining probability will simply be $J$ at any distance. Thus the crossover point at which one term dominates the other is a characteristic vertical length of the network

$$
\xi_{+} \sim \frac{1}{J^{2}}
$$

which can be considered as the typical distance between the 'nodes' of the network; which have at least two completely distinct paths via the network to either the top or the bottom of the system. 
Note, however, that the rejoining probability decays to its long distance value of $J$ only as a power law even for lengths $\gg \xi_{+}$. There thus appears to be no exponential decay of correlations in the flowing phase.

For the one deep model an intersection on the channel network has two incoming and two outgoing channels (for more general models this will not be the case). The definition of 'distinct paths' is that they not intersect at any sites. Thus in the 'braided' structures observed in, e.g., the pair of channels on the right side of Fig. 过, only the top and bottom intersections of the braid between the channels are true nodes. By the usual definitions of percolation theory all of the channel network is part of the 'backbone' while the definition of 'nodes' is the same as here.

Near threshold, intersections between channels will be much more numerous than nodes. The mean distance between intersections along a channel is $\mathrm{O}\left(J^{-1}\right)$, but the characteristic vertical distance between nodes is $\mathrm{O}\left(J^{-2}\right)$ (Eq. 6). In mean field theory the distinction between nodes and intersections is lost and both are spaced by $\mathrm{O}\left(J^{-1}\right)$.

Another property of the steady state channel network that follows immediately from the above picture, is its symmetry: the network will be statistically up-down symmetric. This is not at all obvious from the definition of the model, but is a feature of the steady state. Indeed other features such as the statistics of the 'drainage network' - sites on which an added particle will join the network and fall to the bottom - are definitely not up-down symmetric This is illustrated by Fig. 固 which shows the channel network and Fig. (6) which also shows the drainage network and is clearly not up-down symmetric.

\section{B. Beyond the one-deep model}

We have seen that the simple one-deep model exhibits rather simple correlations in the steady state channel network, and power law approach to the steady state. How do these features persist for more complicated versions of the model?

The two-deep model also has trivial correlations within each row (which depend on the 
parameter $r$ ), with the outlet occupation probabilities being independent as in the mean field approximation. But the inter-row correlations can no longer be treated by the trick used for the one-deep case. For variants, like the natural three-deep generalization (which has three parameters analogous to $r$ of the two-deep model), the outlet occupation probabilities within one row are no longer independent. Nevertheless, we conjecture that for the general synchronous models, the behavior at large scales will be the same as for the one-deep model, with only short range correlations in the outlet occupation probabilities within each row, and power law correlations between rows induced by the diffusion-like behavior of random walkers.

The convergence to the steady state of the two copies in more general models is also more complicated than for the one-deep model. As discussed in the mean field context, the annihilation diffusion representation is now more complicated with several reacting and annihilating species. Nevertheless the experience with reaction diffusion system in other contexts suggests that similar behavior may persist with $t^{-1 / 4}$ decay of the defect density 44

\section{Inter-row Diffusion}

So far we have neglected the possibility of movement of particles between different rows of particles, i.e., between rows with different $y-t$. Each row has been treated independently, every particle moving at the same speed. A more realistic model might involve asynchronous dynamics with different particles moving at different speeds, crudely this can be thought of superimposing some kind of inter-row diffusion on top of the constant velocity motion.

If the different speeds are a result only of the underlying lattice, for instance if particles move through some outlets more quickly, then inter-row motion will be the same in two copies of the system. This means that the $A+B \rightarrow \emptyset$ picture of the approach to equilibrium will survive, with the modification that now the reaction diffusion occurs in all of the spatial dimensions not just the transverse ones. The concentrations will then decay as $t^{-d / 4}$ rather than $t^{-(d-1) / 4}$ so that on our two dimensional lattice we expect decay as $t^{-1 / 2}$. If the inter- 
row motion is slow then there will be a crossover between the $t^{-1 / 4}$ behavior at small scales and $t^{-1 / 2}$ at large scales.

But the inter-row motion might also be affected by the number of particles at each site. A site with many particles might overflow more quickly than one with fewer particles. This type of modification is less easy to accommodate within a reaction diffusion system, but we expect it will produce qualitatively similar behavior at large scales.

\section{Thermal Fluctuations}

It is also possible to consider a very simple finite temperature version of our model within the $A+B \rightarrow \emptyset$ picture. Thermal fluctuations would cause a particle to have some chance of choosing the higher outlet instead of the lower one. This chance would be stochastic and could happen differently in two copies. If a site has one particle in each of the two copies and then, due to thermal fluctuations, moves through different outlets in the two copies then within the $A+B$ picture an unlabeled site will split into an $A$ site which has a particle in the first copy only, and a $B$ site which has a particle only in the second copy. This suggests replacing the reaction $A+B \rightarrow \emptyset$ with the reversible reaction

$$
A+B \rightleftharpoons \emptyset .
$$

in this case the statistical steady state will have a finite concentration of $A$ and $B$ sites controlled by the relative rate of the backward reaction which is determined controlled by the scale of the thermal fluctuations.

\section{BELOW THRESHOLD}

We now turn to an investigation of some of the behavior below threshold. The motion

of particles will now only be transient, but one can also ask about the properties of the stationary state reached when the transients have decayed. 
As discussed in the Introduction, there will be substantial history dependence. If the force is suddenly increased, many excess particles will appear and these can collide, resulting in splits and some particles moving through secondary outlets.

A simpler situation - on which we will focus here - is a slow adiabatic increase in the force such that at any time there are very few excess particles and, in the infinitesimally slow limit, there will be no collisions or splits and every particle will follow a primary outlet path until it finds a trap. The resulting configuration will not depend on the order in which the excess particles appear, thus we can consider starting with all the excess particles in the system and follow the system down from row to row, letting all the excess particle enter a site from higher rows, first filling up the site with any excess then moving all of these out through the primary outlet. We can thus 'sweep' down the system keeping track of the number of excess particles on each site which will then be 'swept' into the row below, picking up some extras from the initial excess particles on the lower row and losing some to unsaturated sites in that row.

We will specifically consider initial conditions in which each site in the lattice has an independently chosen number of particles, $a$, measured relative to the local capacity. Negative values of $a$ represent unsaturated sites (traps) and $a=0$ represents sites that cannot trap particles but have no excess particles. The distribution of values of $a$ will be represented by $A_{a}$. It is convenient to consider the case where $A_{a}$ only has weight for $a \geqslant-1$. Later the particular case

$$
A_{a}=F \delta_{a, 1}+(1-F) \delta_{a,-1}
$$

will be considered in detail, $F$ representing the applied force.

\section{A. Mean Field Equations}

In the mean field approximation, the sites on each row are independent. Thus the distribution of excess particles in the sweep process is entirely determined by the function $p_{y}(\tilde{n})$ 
defined as the probability distribution of the excess number of particles, $\tilde{n} \geqslant 0$, that are on a site in row $y$ after the excess particles from higher rows have moved to the site, but before they have been allowed to move out through the (primary) outlet.

The probability that a site on the next row down has $\tilde{n}$ excess particles can be constructed simply by considering the number of entering particles and the number of particles from the initial conditions. This equation has a simple form for $\tilde{n} \geqslant 1$ (extra terms are needed for $\tilde{n}=0$, see the Appendix)

$$
\begin{aligned}
p_{y+1}(\tilde{n})= & c_{0} A_{\tilde{n}}+c_{1} \sum_{m=-1}^{\tilde{n}} A_{m} p_{y}(\tilde{n}-m)+ \\
& c_{2} \sum_{m=-1}^{\tilde{n}} \sum_{\ell=0}^{\tilde{n}-m} A_{m} p_{y}(\ell) p_{y}(\tilde{n}-m-\ell) .
\end{aligned}
$$

where the terms represent, respectively, sites with zero, one, or two inlets that are primary outlets from the row above. The coefficients $\left\{c_{i}\right\}$ are the probabilities for these numbers of primary inlets; on the square lattice

$$
c_{0}=\frac{1}{4} \quad c_{1}=\frac{1}{2} \quad c_{2}=\frac{1}{4} .
$$

The analysis of Eq. (86) is similar to that carried out for the continuum problem by NF and is described in the Appendix.

Far down from the top, $p_{y}(\tilde{n})$ approaches a limiting distribution $p(\tilde{n})$ which can be computed explicitly. For the specific distribution Eq. (85) for the initial excess particles, it is found that there is a critical value $F=1 / 4$ beyond which no limiting distribution exists. This is the threshold

$$
F_{a}=1 / 4
$$

for adiabatic increase of $F$. Note that this critical force is lower than that $(F=1 / 2)$ for the total number of particles to just fill up all the sites.

At threshold, the limit distribution has a power law tail

$$
p(\tilde{n}) \sim \frac{1}{\tilde{n}^{5 / 2}} .
$$


Since at most one excess particle can be trapped by each row going down the primary outlet path from a site, this power law tail implies that the probability that some of these particles will fall down by at least a distance $y$ before being trapped decays as a power law-although one that is not simply related to that in Eq. (89).

For $F<F_{a}$ the limit distribution decays exponentially, which is related - although very non-trivially due to the pick up of extra particles as the sweep continues downwards - to an exponential tail in the probability that any particle will fall a large distance $y$ before being trapped. This fall-distance probability can be explicitly probed by considering the decay, in moving down the system, of a perturbation in which one extra particle is added; this yields the probability that the extra particles will move down a distance $y$ before stopping. From the Appendix it is found that for large $y$ this decays as

$$
p_{\text {fall }}(y) \sim \frac{1}{y^{3 / 2}} e^{-y / \xi_{-}}
$$

with correlation length $\xi_{-}$that diverges as

$$
\xi_{-} \sim \frac{1}{\left(F_{a}-F\right)^{3 / 2}}
$$

as the threshold is approached, i.e., the critical exponent $\nu_{a}=3 / 2$.

After all particles have come to rest the configuration consists (as discussed in the Introduction and by NF) of saturated trees connected by primary outlets and terminating (at the bottom) in an unsaturated terminus site. An excess particle added anywhere on this tree will fall to the terminus site, possibly thereby extending the tree downwards if it saturates the terminus site. The correlation length Eq. (91) then gives the characteristic vertical length above which larger trees become exponentially rare. On smaller scales than $\xi_{-}$, but near to $F_{a}$ so that $\xi_{-}$is long, the trees are fractal with number of sites scaling with vertical length as $\sim L^{d_{f}}$. In mean field theory, the fractal dimension

$$
d_{f}=\frac{4}{3} .
$$

From calculations analogous to those in the Appendix, and in NF, one can obtain a scaling form for the probability that a site is on a saturated cluster of length $\ell$ : 


$$
\rho(\ell, F) d \ell \sim \frac{1}{\ell^{\kappa_{a}}} \hat{\rho}\left(\ell / \xi_{-}\right) \frac{d \ell}{\ell}
$$

with the scaling function $\hat{\rho}$ decaying exponentially for large argument and, in mean field theory,

$$
\kappa_{a}=\frac{2}{3}
$$

The probability that an added particle will fall a distance $y$ before stopping will have a similar scaling form with the same $\kappa_{a}$ but a different scaling function.

\section{B. Two dimensions}

In our two dimensional system, the transient and saturated trees that form on the adiabatic approach to threshold will be similar to those in the continuum model studied by NF. The distribution of tree lengths will have a form similar to Eq. (93) with power laws out to a vertical distance of order

$$
\xi_{-} \sim \frac{1}{\left(F_{a}-F\right)^{\nu_{a}}}
$$

with the exponents, $\kappa_{a}$ and $\nu_{a}$, and the scaling function $\hat{\rho}$ different from their mean field values. The trees will be fractal with some fractal dimension $d_{f}$. In NF it was asserted that the width of the trees should scale as the square root of the length. This may well not be correct and the more general result

$$
W \sim L^{\alpha_{a}}
$$

should apply. NF estimated the exponents from numerical simulations assuming that $\alpha_{a}=$ 1/2. Their results [Eq. (3a)] may thus be somewhat off, although the consistency of the analysis suggests that $\alpha_{a}$ is probably close to $1 / 2$.

The subtle effect, known for other directed percolation-like models, but missed by NF, is that, although the primary outlet paths are random walks in the transverse direction, those outlet paths that wander anomalously far typically will have a different area from 
which they can collect excess particles than outlet paths that go closer to straight downhill. This should make the likelihood of wider trees grow differently than that of narrower ones resulting in the possibility of changing the scaling of the large trees and yielding $\alpha_{a} \neq 1 / 2$.

In our system, however, there is perhaps some reason to suspect that $\alpha_{a}$ could be exactly $1 / 2$. Above threshold, the results on the channel network yield that the exponent, $\alpha_{+}$, which relates scaling in the vertical and transverse directions is exactly $1 / 2$. If there is some form of two-sided scaling, as suggested in section $\square$, then features above threshold (e.g., finite trees disconnected from the channel network) would be expected to scale, for intermediate length scales, in similar ways to those below threshold, i.e., with $\alpha_{a}$. How $\alpha_{a} \neq 1 / 2$ could fit into a two-sided scaling picture with the channel network with $\alpha_{+}=1 / 2$ is unclear; the simplest scenarios would suggest, therefore, that $\alpha_{a}=\alpha_{+}=1 / 2$.

Further numerical simulations in two dimensions should be able to resolve this, although large sizes and good statistics will be needed.

In any case, with general $\alpha_{a}$, the scaling law of NF relating $d_{f}$ and $\kappa_{a}$ in two dimensions should be replaced by

$$
d_{f}=1+\alpha_{a}-\kappa_{a}
$$

\section{TRANSIENTS AND HISTORY DEPENDENCE}

In the previous two sections, we have seen that our model has two simple limits: below threshold when the force is increased adiabatically for which no splits occur, and above threshold at constant current so that the unsaturated sites play no role if the focus is just on the steady state river network. In both these simple limits, a crucial part of the physics

plays no role, simplifying the behavior but yielding, not surprisingly, scaling properties for the two phases which appear to be totally unrelated.

In this section we raise some issues and make a few observations about the largely unexplored regimes between these two limits. We will focus on the behavior in an infinite system 
starting from an initial condition corresponding to the force to be studied, or correspondingly, the 'sudden' limit where the force is suddenly increased from zero (i.e., from having no excess particles) to a particular value. As long as the time to reach steady state in the bulk is much less than the transit time of particles from top to bottom, the same behavior should appear also in finite systems except in a growing boundary region near the top; however in contrast to the situation studied in Sec. III, the current will be determined in a non-trivial way from the initial distribution of excess particle and unsaturated sites.

One might hope to use mean field theory to obtain results for this situation. Unfortunately the tricks which made mean field theory tractable for the cases discussed in the previous sections - the following of individual rows or sets of rows in one pass down through the system - fail here. This is because whether a particle gets trapped at a site will depend on the previous history of the site. Thus the inputs to a site from the row above, while independent from each other, are correlated with the inputs at earlier times. So far, we have been unable to derive interesting results from mean field theory for the sudden limit.

We thus restrict ourselves in this section to qualitative discussions and general scaling arguments.

\section{A. Below Threshold}

As discussed in the Introduction, the behavior as the force is suddenly increased is rather different from the adiabatic behavior discussed in Sec. IV. Initially, there will be a large number of collisions and splits so that some of the particles will follow secondary outlets and fill unsaturated sites that would not have been filled with an adiabatic increase in force.

We can characterize the transients and the final stationary state in various ways. At long times, there will be a small density of particles still moving and, even though these will tend to be collected together by their motion downhill, involving mostly primary outlets, the loss of particles to traps will dominate for $F$ less than some critical value $F_{s}\left(>F_{a}\right)$, and the last moving particles will follow paths similar to those in an adiabatic approach. Near $F_{s}$, 
we might expect a power law decay of moving particles at intermediate times, cutoff by an exponential decay at long times with a characteristic time scale that diverges at $F_{s}$.

The stationary state can be characterized in a similar way to the adiabatic case, by the distribution of saturated trees - connected by primary outlets - which have the property that an extra particle which is added on a tree will fall to the (unsaturated) terminus site before stopping. These trees, as in the adiabatic case, will be subsets of the complete primary outlet tree which connects all sites. But they will, in general, be different subsets due to the splitting processes, and for a given $F$ will tend to be smaller. Again, near $F_{s}$, we expect a distribution of saturated tree lengths of similar form to Eq. (93) but with possibly different exponents $\kappa_{s}, \nu_{s}$ and scaling of the widths with the length of clusters of $W \sim L^{\alpha_{s}}$. The relation between the number of sites in the large clusters to their length should again be given by a fractal dimension related to $\alpha_{s}$ and $\kappa_{s}$ by Eq. (97).

An outstanding open question is whether or not, in fact, the sudden approach to threshold is a different universality class from the adiabatic approach. One might expect this to depend on the rate of collisions and splits at times of order $\xi_{-}$near threshold. This should also affect whether the relaxation time scales simply as $\xi_{-}$(as in the adiabatic case) or is much longer due to the splits. We leave these issues for future study.

Another property of the stationary final state is the fraction of unsaturated sites, which is equal to the number density of saturated trees (since these include trivial 'trees' that consist of only a single 'terminus' site). This is clearly non-zero below $F_{s}$, but, a priori, it could in principle approach zero at threshold if the exponent $\kappa_{s}$ is zero so that the large saturated trees contain most of the sites and are not really fractal, i.e., $L^{d_{f}} \sim L \times L^{\alpha_{s}}$. This question is related to the issue of unsaturated sites above threshold; the arguments below strongly suggest that there will be a finite density of unsaturated sites both at threshold and above. 


\section{B. Above Threshold}

Above threshold, various aspects of the system started suddenly from an initial configuration that includes unsaturated sites will be different from the constant current driven system discussed in Sec. [II].

One set of properties will, however, be the same. At long times, the system will have a good separation between the moving and stationary parts of the system and, by the arguments of Sec. III, the channel network will become asymptotically time independent with the steady state network the same as that for the current driven case far from the top boundary at the same current density. (Note that the approach to this network will involve extra splits and filling in of extra off-network sites resulting in the loss of moving particles, but the basic property of the moving phase is that these processes will, at long times, leave a finite fraction of the particles till moving.)

As mentioned in the Introduction, the dependence of the current density on $F$ will be non-trivial, presumably growing as a power of $F-F_{s}$ near the threshold as in Eq. (7).

With the system evolving from initial conditions to a steady state current network, we must right away consider the role of unsaturated sites. If all the sites eventually become saturated (as in the current driven case with random time dependent currents inserted at the top), then the current would be trivially related to the initial particle density with $F_{s}$

the point at which the mean excess is zero and $J \propto\left(F-F_{s}\right)$. We will now argue that this will in fact, not be the case: some fraction of sites will always remain unsaturated in the infinite-time steady state.

We focus on one site, say the origin, and consider the probability that it never has a particle pass through it. This is clearly underestimated by the case with no unsaturated sites and we thus consider this case; with initial conditions of an excess number of particles distributed randomly with density $J$ among the sites in the one-deep model, so that in the first time step a fraction $J / 2$ of the outlets carry one particle.

An initial configuration in the row a distance $h$ above the origin (i.e., at coordinate $y=$ 
$-h$ ) which has $h$ consecutive doubly occupied sites centered at $x=0$, will ensure that some particle will definitely reach the origin. But the probability of this extreme event is $e^{-c h}$ so that the probability of it not occuring for any $h$ is non-zero if $c$ is large enough. Thus the mechanism which results in filling in all the sites that are a finite distance from the top in the case of a random time dependent current inserted at the top, will not apply here.

But of course, most sites can be reached by less extreme initial conditions. We must therefore rely on the convergence properties of different initial rows passing through the same region as discussed in Sec. [II].

In particular, consider the $z$ rows starting distances between $h$ and $h+z$ above the origin. As these pass through height $h$, there will already be substantial correlations in the position of the moving particles and ignoring these will tend to overestimate the probability of reaching the origin; thus in order to underestimate the probability, we consider these $z$ rows as starting with independent random configurations - $z$ 'copies' - at height $h$.

From the aggregation analogy in Sec. III, the simplest guess is that in each region of width $\sim \sqrt{h}$ the copy with most particles in it will essentially 'capture' all the particles in the other copies, so that the positions of the particles in the other copies at row zero will be subsets of the positions of the dominant copy in that region. Since the copy with most particles in a region of width $\sqrt{h}$ will have

$$
J \sqrt{h}+\mathrm{O}\left(h^{1 / 4} \sqrt{\ln z}\right)
$$

particles, this suggests that a fraction of order $h^{-1 / 4} \sqrt{\ln z}$ of row zero that is not on the steady state network will be reached by some particle from these $z$ rows. By choosing, e.g., $z=h$ and considering the set of rows with $h=1,2,4,8, \ldots 2^{k}, \ldots$, we would conclude that the total fraction $f_{R}$ of off-network sites reached from any of these sets of initial rows is

$$
f_{R}<C \sum_{k=1}^{\infty} 2^{-k / 4} \sqrt{k},
$$

which is less than one providing $C$ is sufficiently small; it will be so for small $J$. For large $J$ 
more care is needed in the estimate of the effects of nearby rows, but the quantitative mechanism will still obtain.

This argument is, unfortunately, not quite correct. Because of the splittings, the majority copy will not, in general 'capture' all of the other copies in a region; and thus Eq. (98) will be an underestimate of the fraction of sites reached. But for the series in Eq. (99) to converge we need only a much weaker estimate. The physics involved in the tendency of the rows from far above to arrive at the origin looking similar should, we believe, make a better argument along the above lines possible. But we leave the necessary work for the future and, at this point, just pose as a conjecture that a finite fraction of the sites will remain unsaturated in the steady state. Preliminary numerical studies strongly support this conjecture.

If this conjecture is correct, then various properties of the off-network sites become interesting. In particular, if an excess particle is added to the system in the steady state, it will have only a finite probability $P_{\infty}$ of moving onto the channel network and hence down to the bottom of the system. We conjecture that near threshold the fraction of sites in the 'drainage network', $P_{\infty}$, behaves as a power law of $F-F_{s}$ by analogy with percolation:

$$
P_{\infty} \sim\left(F-F_{s}\right)^{\Gamma_{s}}
$$

The statistics of the drainage network on scales smaller than the distances between nodes

on the channel network- $\xi_{+}$vertically, $\sim J^{-1}$ in transverse directions - should be related to the statistics of saturated trees below threshold; providing a link between above and below threshold scaling. Note however the subtleties raised at the end of section IV if $\alpha \neq 1 / 2$. We leave analysis of the scaling behavior and the decay of transients in the moving phase for future work.

\section{Adiabatic History}

We close this section with a brief discussion of issues associated with the alternate, adiabatic history analyzed in Sec. IV below threshold. 
If the force is increased at a slow but finite rate, just below the adiabatic threshold, new excess particles will appear fast enough that, as they collect on the primary outlet tress, collisions will become likely resulting in splits. But once the number of particles becomes sufficiently large above the adiabatic threshold, the moving particles should be able to establish a steady state channel network on time scales slower than that characterizing the rate of adding more excess particles, i.e., the rate of increase of $F$.

Near $F_{a}$, there will be non-adiabatic effects, but in the limit of zero rate of increase of $F$, it would appear that collisions that are not present in the steady state channel network will become rare. Thus we conjecture that in this adiabatic limit, no sites will be filled except those on the steady state channel network at the final force $F>F_{a}$, and those that can be reached by primary outlet paths (i.e., without splits).

Even if this conjecture is not correct, it appears that the adiabatic history should yield an alternate steady state above threshold with a drainage network whose statistics should be related to those of the saturated trees in the adiabatic history below threshold analyzed in Sec. IV. Again we leave study of these and other properties, which should be related to those of the continuous fluid discussed by NF, for the future.

\section{APPLICATIONS AND CONCLUSIONS}

We have seen that a very simple model of driven particle motion in a random system yields very rich behavior, including the development of distributions of event sizes and fractal spatial structures that are precursors to steady state flow which occurs, just above a threshold drive, on a sparse network of channels with most of the system stationary.

There are various possible applications of at least the qualitative features of our results.

\section{A. Magnetic Bubbles}

The system of magnetic bubbles in a film driven by a magnetic field gradient, as studied

by Westervelt and collaborators, 23,47 is the simplest potential application. The model is 
most directly applicable in the presence of a high density of strong pinning centers for the bubbles. The main complication is the effect of the positions of bubbles in a 'trap' on other particles, in particular on how they exit a trap. If these effects are small, then many of our results should be directly observable; for example the statistics of the distances particles move on increasing the drive below threshold, and the reproducibility and statistics of the channel network above threshold. Experiments have not yet been performed in this regime but seem feasible.

For weaker or more dilute pinning, the bubbles will form regions of hexagonal crystal. On longer scales, however, the strains built up by the drive through the random medium should be sufficient to break up the lattice, as pointed out by Coppersmith. 36 The sizes of these micro-crystallites can then crudely be thought of as setting the width of channels; just above the threshold drive, most will stay still, and motion should occur primarily along a sparse network of channels. Because of the effect of the bubbles on each other in this regime the network will probably be more history dependent than in our models and may evolve somewhat in time as regions reorient, grain boundaries move, etc. However the qualitative aspects of broadly distributed effects of increases in the force below threshold and a sparse channel network above threshold, as well as history dependence of the threshold force, should persist.

\section{B. Thin Film Superconductors}

The most interesting application is vortex dynamics in thin film superconductors. But in addition to the effects mentioned above for magnetic bubbles, there are other complications. If the film is thick, the vortices will not behave as two-dimensional points, but as lines bringing in much interesting new physics. If the film is thin, on the other hand, the interactions between the vortices will be long range-logarithmic out to the transverse penetration length $\left(\Lambda_{\perp}=2 \lambda^{2} / d\right.$ with $d$ the thickness and $\lambda$ the bulk penetration length), and $1 / r$

at large distances. For samples small compared to $\Lambda_{\perp}$, the applied current density will be 
relatively uniform and the applied forces on the vortices roughly equal. For larger films, the current will initially flow primarily at the edges and only penetrate further concomitantly with the vortex density building up or being depleted. This effect should not be too large if the magnetic fields due to the applied current are small compared to the mean magnetic field, but can play a role in some regimes. In this regime the vortex density will readjust as the current is increased to yield roughly constant forces on the vortices near the critical current. How this will effect the various features of our model is unclear.

The regime of most direct applicability is a film with holes that in equilibrium will contain all the vortices. The configuration in a hole will then only depend on the number of vortices in it, although the direction in which vortices will be pulled from a hole will in general depend on the occupation of other holes. Nevertheless, many of the predictions of the model may be rather directly testable, although there may be extra history dependence and other modifications due to the long-range interactions.

The first descriptions of the channel flow of flux lines in thin film superconductors were

numerical simulations performed by Berlinsky, Brass, Brecht, and Jensen.21,22,48, 49, Although quantitative comparison with our results is not possible at this point, their qualitative description of 'pulsating flow' along well-defined channels with the majority of the flux lines remaining trapped is the same as ours. Earlier simulations of this system by Brandt50 had extensively studied the weak pinning regime where the Larkin-Ovchinnikov description of the flux lines as an elastic lattice was appropriate, but did not investigate how the lattice broke up for higher pinning strengths.

\section{ACKNOWLEDGMENTS}

We thank A. J. Berlinsky, E. Domany, J. Krim, A. A. Middleton, O. Narayan, S. Redner, and M. S. Tomassone for useful discussions. This work was supported by the National Science Foundation via DMR 9106237 and the Harvard University Materials Research Science and Engineering Center. 


\section{APPENDIX: MEAN FIELD THEORY BELOW THRESHOLD}

This mean field calculation for discrete particles has similar structure to that for con-

tinuous systems. 30 This similarity contrasts with the situation above threshold where the discrete and continuous cases are qualitatively different.

The recursion relation for the probability distribution of the excess number of particles $(\tilde{n})$ at a site in row $y$, for $\tilde{n} \geqslant 1$ is [Eq. (86)]

$$
\begin{aligned}
p_{y+1}(\tilde{n})= & c_{0} A_{\tilde{n}}+c_{1} \sum_{m=-1}^{\tilde{n}} A_{m} p_{y}(\tilde{n}-m)+ \\
& c_{2} \sum_{m=-1}^{\tilde{n}} \sum_{\ell=0}^{\tilde{n}-m} A_{m} p_{y}(\ell) p_{y}(\tilde{n}-m-\ell) .
\end{aligned}
$$

For $\tilde{n}=0$ we have to add the extra terms

$$
c_{0} A_{-1}+c_{1} A_{-1} p_{y}(0)+c_{2} A_{-1} p_{y}^{2}(0)
$$

to the right hand side of Eq. (A1), to account for cases when the site is left below capacity since these also correspond to $\tilde{n}=0$. On the square lattice the probabilities for the numbers of inlets are

$$
c_{0}=\frac{1}{4} \quad c_{1}=\frac{1}{2} \quad c_{2}=\frac{1}{4}
$$

Defining transforms

$$
\begin{aligned}
& p_{y}(\omega)=\sum_{\tilde{n}=0}^{\infty} p_{y}(\tilde{n}) e^{i \omega \tilde{n}} \\
& A(\omega)=\sum_{a=-1}^{\infty} A_{a} e^{i \omega a}
\end{aligned}
$$

the recursion equations become

$$
\begin{aligned}
p_{y+1}(\omega)= & \frac{1}{4} A(\omega)\left[1+p_{y}(\omega)\right]^{2}+ \\
& \frac{1}{4} A_{-1}\left(1-e^{-i \omega}\right)\left(1+\Pi_{y}\right)^{2}
\end{aligned}
$$

where 


$$
\Pi_{y} \equiv p_{y}(n=0)
$$

gives the fraction of sites in row $y$ that have no excess particles.

The fixed point condition $p_{y+1}=p_{y}=p$ has two solutions for $p(\omega)$,

$$
\frac{2-A(\omega) \pm \sqrt{4[1-A(\omega)]-A_{-1}\left(1-e^{-i \omega}\right) A(\omega)(1+\Pi)^{2}}}{A(\omega)}
$$

If we examine the limit $\omega \rightarrow 0$, we see that the argument of the square root has a root at $\omega=0$. Such a single root will give a divergence in $d p / d \omega$ at $\omega=0$ which corresponds to an infinite mean number of particles. This cannot happen below threshold. To avoid this the root at $\omega=0$ must be a double root which requires

$$
\left.\frac{d}{d \omega}\left\{4[1-A(\omega)]-A_{-1}\left(1-e^{-i \omega}\right) A(\omega)(1+\Pi)^{2}\right\}\right|_{\omega=0}
$$

to be equal to zero, which means that $\Pi$ must satisfy

$$
(1+\Pi)^{2} A_{-1}=-4 \bar{a}
$$

with $\bar{a}=\sum_{a} a A_{a}$ the mean number of excess particles. Thus $\bar{a}$ must be negative up to, and including, threshold.

Specializing to the case $A(\omega)=F e^{i \omega}+(1-F) e^{-i \omega}$ yields the solution

$$
\begin{aligned}
p(\omega) & =\frac{F-1+2 e^{i \omega}-F e^{2 i \omega}}{1-F+e^{2 i \omega} F} \\
& -\frac{2\left(e^{i \omega}-1\right) \sqrt{(1-F)\left(1-2 F-2 e^{i \omega} F\right)}}{1-F+e^{2 i \omega} F}
\end{aligned}
$$

As $\omega \rightarrow 0$ this expression has branch cuts at $F=1$ and at $F=1 / 4$. Identifying the latter as the adiabatic critical point $F_{a}=1 / 4$ we have at criticality,

$$
p_{c}(\omega)=\frac{-3+8 e^{i \omega}-e^{2 i \omega}+2 \sqrt{6}\left(1-e^{i \omega}\right)^{3 / 2}}{3+2 e^{i \omega}} .
$$

This has a $\omega^{3 / 2}$ singularity as $\omega \rightarrow 0$ which means the critical distribution of excess particle numbers in the sweep behaves as

$$
p(\tilde{n}) \sim \frac{1}{\tilde{n}^{5 / 2}}
$$


for large $\tilde{n}$.

As we approach the threshold distribution from below we must thus expect the variance of the $p(\tilde{n})$ distribution to diverge while the mean remains finite. Calculating explicitly for

$$
f \equiv F_{a}-F
$$

we find

$$
\begin{aligned}
\langle\tilde{n}\rangle & =1-2 \sqrt{3} f^{1 / 2}+\mathrm{O}\left(f^{3 / 2}\right) \\
\left\langle\tilde{n}^{2}\right\rangle-\langle\tilde{n}\rangle^{2} & \sim \frac{\sqrt{3}}{4} \frac{1}{f^{1 / 2}}
\end{aligned}
$$

which have the same exponents as the continuous fluid studied by NF.

The steady state mean field calculation gives information about the distribution of particle occupations. It could be modified as in the continuous fluid case to provide the distribution of connected cluster sizes. 30 However to obtain information about time scales (or, equivalently, cluster sizes in the downhill direction) it is necessary to consider a non-equilibrium calculation.

If the recursion relation between $p_{y+1}(\omega)$ and $p_{y}(\omega)$ [Eq. (A6)] is considered for distributions that differ from the fixed point value $p(\omega)$ by a small perturbation $\delta p_{y}(\omega)$ then to linear order in the perturbation

$$
\begin{aligned}
\delta p_{y+1}(\omega)= & \frac{1}{2} A(\omega)[1+p(\omega)] \delta p_{y}(\omega)+ \\
& \frac{1}{2} A_{-1}\left(1-e^{i \omega}\right) \delta \Pi_{y}
\end{aligned}
$$

where $\delta \Pi_{y}$ is the perturbation of $p_{y}(x=0)$ away from its fixed point value.

With a perturbation at an initial row $y=0$, the recursion relation is simplified by considering the transforms

$$
\delta p(\omega, z)=\sum_{y=0}^{\infty} \delta p_{y}(\omega) z^{y}
$$

and 


$$
\delta \Pi(z)=\sum_{y=0}^{\infty} \delta \Pi_{y} z^{y}
$$

We can now solve for $\delta p(\omega, z)$ yielding

$$
\delta p(\omega, z)=\frac{\delta p(\omega, 0)+\frac{1}{2} z A_{-1}\left(1-e^{-i \omega}\right) \delta \Pi(z)}{1-\frac{1}{2} z A(\omega)[1+p(\omega)]}
$$

where to be self consistent $\delta p(\omega, 0)$ must also be given by the integral around the unit circle

$$
\delta p(\omega, 0)=\frac{1}{2 \pi i} \oint \frac{\delta p(\omega, z)}{z} d z
$$

For the specific case $A(\omega)=F e^{i \omega}+(1-F) e^{-i \omega}$, Eq. (A19) becomes

$$
\delta p(\omega, z)=\frac{\delta p(\omega, 0)+\frac{1}{4} z\left(1-e^{-i \omega}\right) \delta \Pi(z)}{1-z+z\left(1-e^{-i \omega}\right) \sqrt{(1-F)\left[1-2 F\left(1+e^{i \omega}\right)\right]}}
$$

which has poles at $z=0$ and at $z=z(\omega)$ where

$$
z(\omega)=\frac{1}{1+\left(1-e^{-i \omega}\right) \sqrt{(1-F)\left[1-2 F\left(1+e^{i \omega}\right)\right]}}
$$

Performing the contour integral we have the self-consistency condition

$$
\delta \Pi(z)=\frac{-2 \delta p[\omega(z), 0]}{z A_{-1}\left(1-e^{-i \omega z}\right)}
$$

where $\omega(z)$ is the inverse of the $z(\omega)$ function. We must find the singular parts of this function which dominate the long time behavior of the inverse transform of $\delta \Pi(z)$.

Now $z(\omega)$ is equal to 1 at $\omega=0$ and at $e^{i \omega}=(1-2 F) / 2 F$. Close to threshold - small $f$ this second value is

$$
\omega=-8 i f+\mathrm{O}\left(f^{2}\right)
$$

In between these two points on the negative imaginary axis, $z(\omega)$ has a simple maximum at

$$
\omega=\omega_{c}=-\frac{16}{3} i f+\mathrm{O}\left(f^{2}\right)
$$

In this vicinity

$$
z=z_{c}+C\left(\frac{\omega-\omega_{c}}{i}\right)^{2}
$$


and so $\omega(z)$ has a branch cut:

$$
\omega=\omega_{c}+i \frac{1}{\sqrt{C}} \sqrt{z-z_{c}}
$$

where for small $f$

$$
z_{c}=1+\frac{16}{3} f^{3 / 2}+\mathrm{O}\left(f^{5 / 2}\right)
$$

and

$$
C=\frac{9}{32} \frac{1}{f^{1 / 2}}+\mathrm{O}\left(f^{1 / 2}\right)
$$

This means that for large $y$ the inverse transform is dominated by this square root branch cut

$$
\delta \Pi_{y} \sim \frac{f^{1 / 4}}{y^{3 / 2}} \exp \left[-4 y\left(\frac{2 f}{3}\right)^{2 / 3}\right]
$$

which has the the form

$$
\delta \Pi_{y} \sim \frac{f^{1 / 4}}{y^{3 / 2}} e^{-y / \xi_{-}}
$$

where the vertical length scale, $\xi_{-}$, diverges at threshold as

$$
\xi_{-} \sim f^{-3 / 2}
$$

The $\nu_{a}=3 / 2$ correlation length exponent for the cluster size (or for the response to perturbations) does not correspond to any of those seen above threshold. It does correspond to that found for the continuous fluid below threshold by NF. 


\section{REFERENCES}

${ }^{1}$ B. Koiller, H. Ji, and M. O. Robbins, Phys. Rev. B 46, 5258 (1992).

${ }^{2}$ H. Ji and M. O. Robbins, Phys. Rev. B 46, 14519 (1992).

3 T. Nattermann, S. Stepanow, L.-H. Tang, and H. Leschhorn, J. Phys. (Paris) II 2, 1483 (1992).

${ }^{4}$ O. Narayan and D. S. Fisher, Phys. Rev. B 48, 7030 (1993).

${ }^{5}$ See, for example, Charge Density Waves in Solids, Vol. 217 of Lecture Notes in Physics, edited by H. Araki et al. (Springer-Verlag, Berlin, 1985) and references therein.

${ }^{6}$ D. S. Fisher, Phys. Rev. Lett. 50, 1486 (1983); Phys. Rev. B 31, 1396 (1985).

${ }^{7}$ A. A. Middleton and D. S. Fisher, Phys. Rev. Lett. 66, 92 (1991); Phys. Rev. B 47, 3530 (1993).

${ }^{8}$ O. Narayan and D. S. Fisher, Phys. Rev. Lett. 68, 3615 (1992); Phys. Rev. B 46, 11520 (1992).

${ }^{9}$ O. Narayan and A. A. Middleton, Phys. Rev. B 49, 244 (1994).

${ }^{10}$ A. I. Larkin and Y. N. Ovchinnikov, J. Low Temp. Phys. 34, 409 (1979).

${ }^{11}$ G. Blatter et al., Rev. Mod. Phys. 66, 1125 (1994).

12 R. Lenormand, J. Phys.: Condes. Matter 2, SA79 (1990).

${ }^{13}$ M. A. Rubio, C. A. Edwards, A. Dougherty, and J. P. Gollub, Phys. Rev. Lett. 63, 1685 (1989); M. A. Rubio, A. Dougherty, and J. P. Gollub, ibid. 65, 1389 (1990).

${ }^{14}$ V. K. Horáth, F. Family, and T. Viscek, J. Phys. A 24, L25 (1991).

${ }^{15}$ S. He, G. L. M. K. S. Kahanda, and P.-z. Wong, Phys. Rev. Lett. 69, 3731 (1992).

${ }^{16}$ C. S. Nolle, B. Koiller, N. Martys, and M. O. Robbins, Phys. Rev. Lett. 71, 2074 (1993). 
17 J. F. Joanny and M. O. Robbins, J. Chem. Phys. 92, 3206 (1990).

${ }^{18}$ D. Ertaş and M. Kardar, Phys. Rev. E 49, R2532 (1994).

${ }^{19}$ A.-L. Barabási and H. E. Stanley, Fractal Concepts in Surface Growth (Cambridge University Press, Cambridge, 1995).

${ }^{20}$ J. Zhang, Y.-C. Zhang, P. Alstrøm, and M. T. Levinsen, Physica A 189, 383 (1992).

${ }^{21}$ H. J. Jensen, A. Brass, and A. J. Berlinsky, Phys. Rev. Lett. 60, 1676 (1988).

${ }^{22}$ H. J. Jensen, A. Brass, Y. Brecht, and A. J. Berlinsky, Phys. Rev. B 38, 9235 (1988).

${ }^{23}$ R. Seshadri and R. M. Westervelt, Physica D 66, 223 (1993).

${ }^{24}$ R. Lenormand and C. Zarcone, Phys. Rev. Lett. 54, 2226 (1985).

${ }^{25}$ M. Cieplak and M. O. Robbins, Phys. Rev. Lett. 60, 2042 (1988).

${ }^{26}$ N. Martys, M. Cieplak, and M. O. Robbins, Phys. Rev. Lett. 66, 1058 (1991).

${ }^{27}$ N. Martys, M. O. Robbins, and M. Cieplak, Phys. Rev. B 44, 12294 (1991).

${ }^{28}$ B. Koiller, H. Ji, and M. O. Robbins, Phys. Rev. B 45, 7762 (1992).

${ }^{29}$ A. P. Kushnick, J. P. Stokes, and M. O. Robbins (unpublished).

${ }^{30}$ O. Narayan and D. S. Fisher, Phys. Rev. B 49, 9469 (1994).

${ }^{31}$ M. S. Tomassone and J. Krim (unpublished).

32 A. A. Middleton and N. S. Wingreen, Phys. Rev. Lett. 71, 3198 (1993).

${ }^{33}$ L. Niemeyer, L. Pietronero, and H. J. Wiesmann, Phys. Rev. Lett. 52, 1033 (1984).

${ }^{34}$ D. Domínguez, Phys. Rev. Lett. 72, 3096 (1994).

${ }^{35}$ K. Harada et al., Phys. Rev. Lett. 71, 3371 (1993).

${ }^{36}$ S. N. Coppersmith, Phys. Rev. Lett. 65, 1044 (1990). 
${ }^{37}$ We thank E. Domany for pointing out this possibility.

${ }^{38}$ S. Chandrasaker, Rev. Mod. Phys. 15, 1 (1943).

${ }^{39}$ B. I. Halperin, S. Feng, and P. N. Sen, Phys. Rev. Lett. 54, 2391 (1985); S. Feng, B. I. Halperin, and P. N. Sen, Phys. Rev. B 35, 197 (1987).

${ }^{40}$ A. A. Ovchinikov and Y. B. Zeldovich, Comm. Phys. 28, 214 (1978).

${ }^{41}$ D. Toussaint and F. Wilczek, J. Chem. Phys. 78, 2642 (1983).

${ }^{42}$ M. Bramson and J. L. Lebowitz, Phys. Rev. Lett. 61, 2397 (1988).

${ }^{43}$ M. Bramson and J. L. Lebowitz, J. Stat. Phys. 65, 941 (1991).

${ }^{44}$ B. P. Lee and J. Cardy, Phys. Rev. E 50, R3287 (1994).

${ }^{45}$ B. P. Lee and J. Cardy, J. Phys. A 27, 2633 (1994).

${ }^{46}$ L. Peliti, J. Phys. A 19, L365 (1986).

${ }^{47}$ J. Hu and R. M. Westervelt, Phys. Rev. B 51, 17279 (1995).

${ }^{48}$ A. Brass, H. J. Jensen, and A. J. Berlinsky, Phys. Rev. B 39, 102 (1989).

${ }^{49}$ H. J. Jensen, A. Brass, Y. Brecht, and A. J. Berlinsky, Cryogenics 29, 367 (1989).

${ }^{50}$ E. H. Brandt, Phys. Rev. Lett. 50, 1599 (1983); J. Low Temp. Phys. 53, 41 (1983); 53, 71 (1983). 


\section{FIGURES}

FIG. 1. Schematic of the lattice model. Circles represent lattice sites and arrows the outlets connecting sites. Thicker lines are primary outlets. The coordinate system is also shown.

FIG. 2. Saturated sites below threshold showing the tree structures. Filled circles represent saturated sites, unfilled circles represent unsaturated terminus sites, and lines show the primary outlets from saturated sites.

FIG. 3. Steady state channel network from an illustrative simulation of the one-deep model with periodic boundary conditions.

FIG. 4. Route Picture. Lines show routes followed by particles. The pattern is specific to a particular row of particles moving down through the system. The choice of 'crossed' or 'uncrossed' at each site depends on the particle distribution within the row, and is used only for computation of single row properties and correlations.

FIG. 5. One-deep model above threshold, with periodic boundary conditions. Lines show those outlets which carry particles in the steady state. Filled circles represent saturated sites, unfilled circles represent unsaturated sites.

FIG. 6. Drainage network corresponding to the channel network shown in Fig. 5. Thick lines show the channel network (outlets through which there is flow in the steady state) and thin lines show the drainage network which links sites for which an added particle will reach the channel network). 


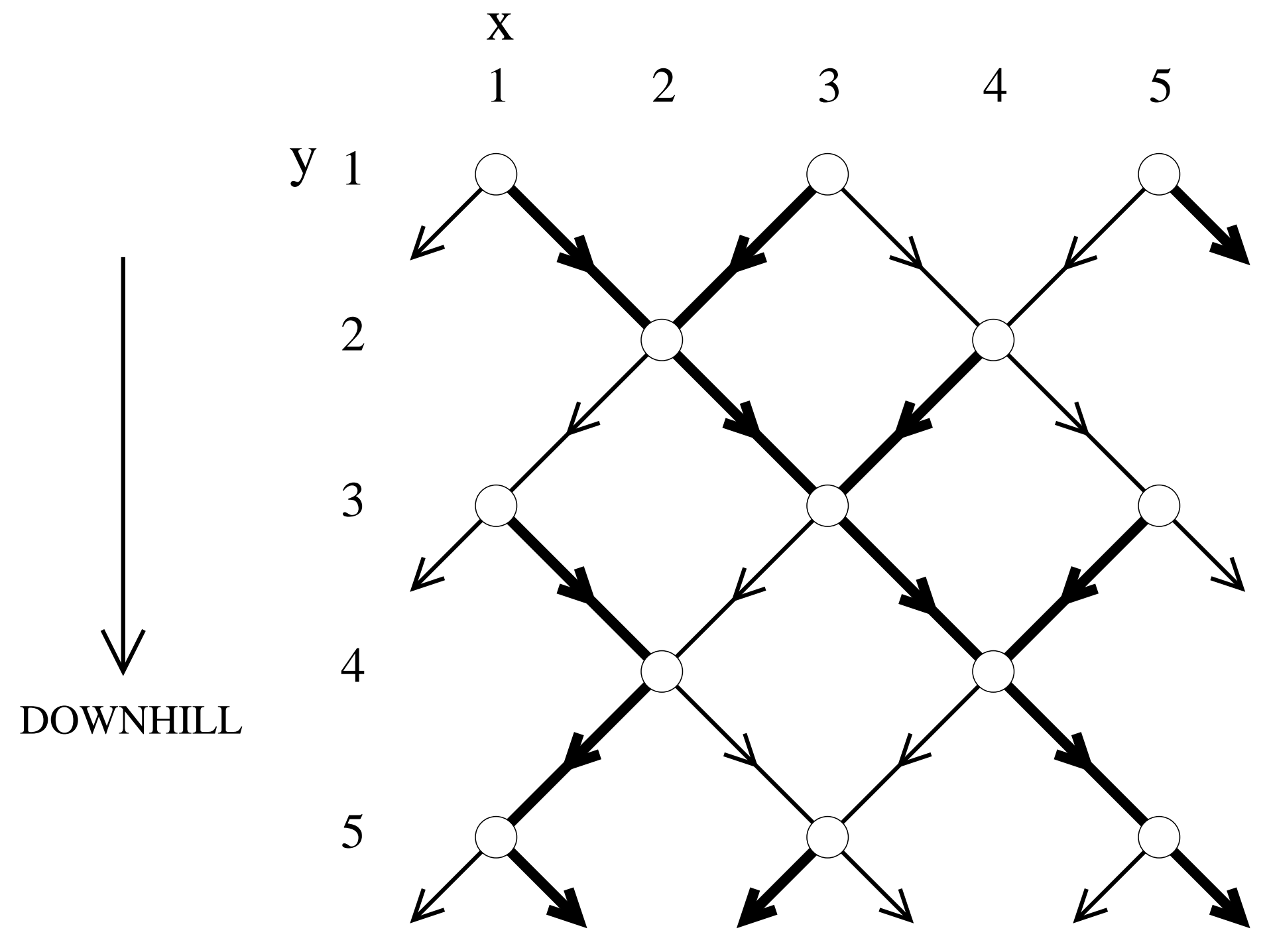



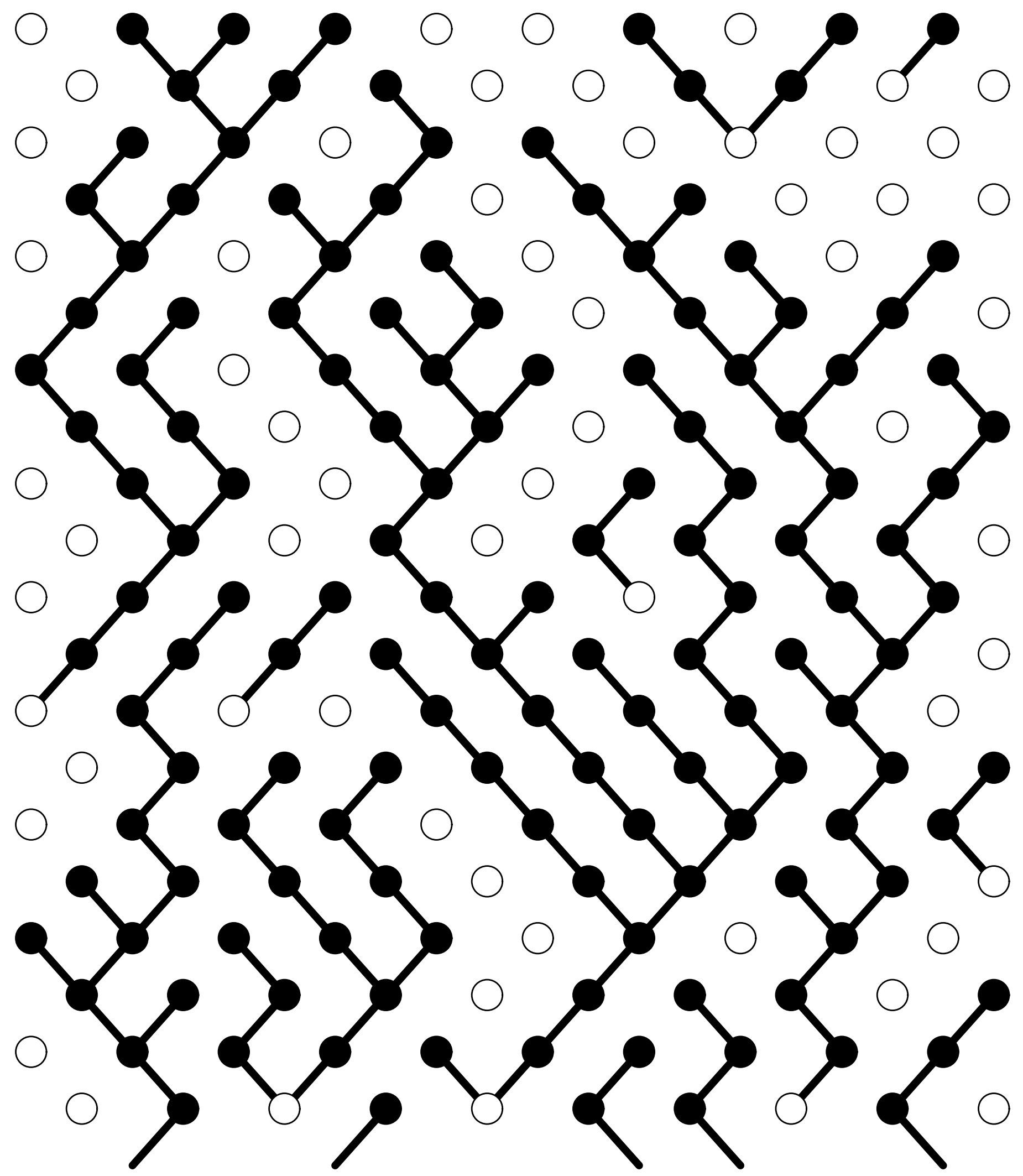


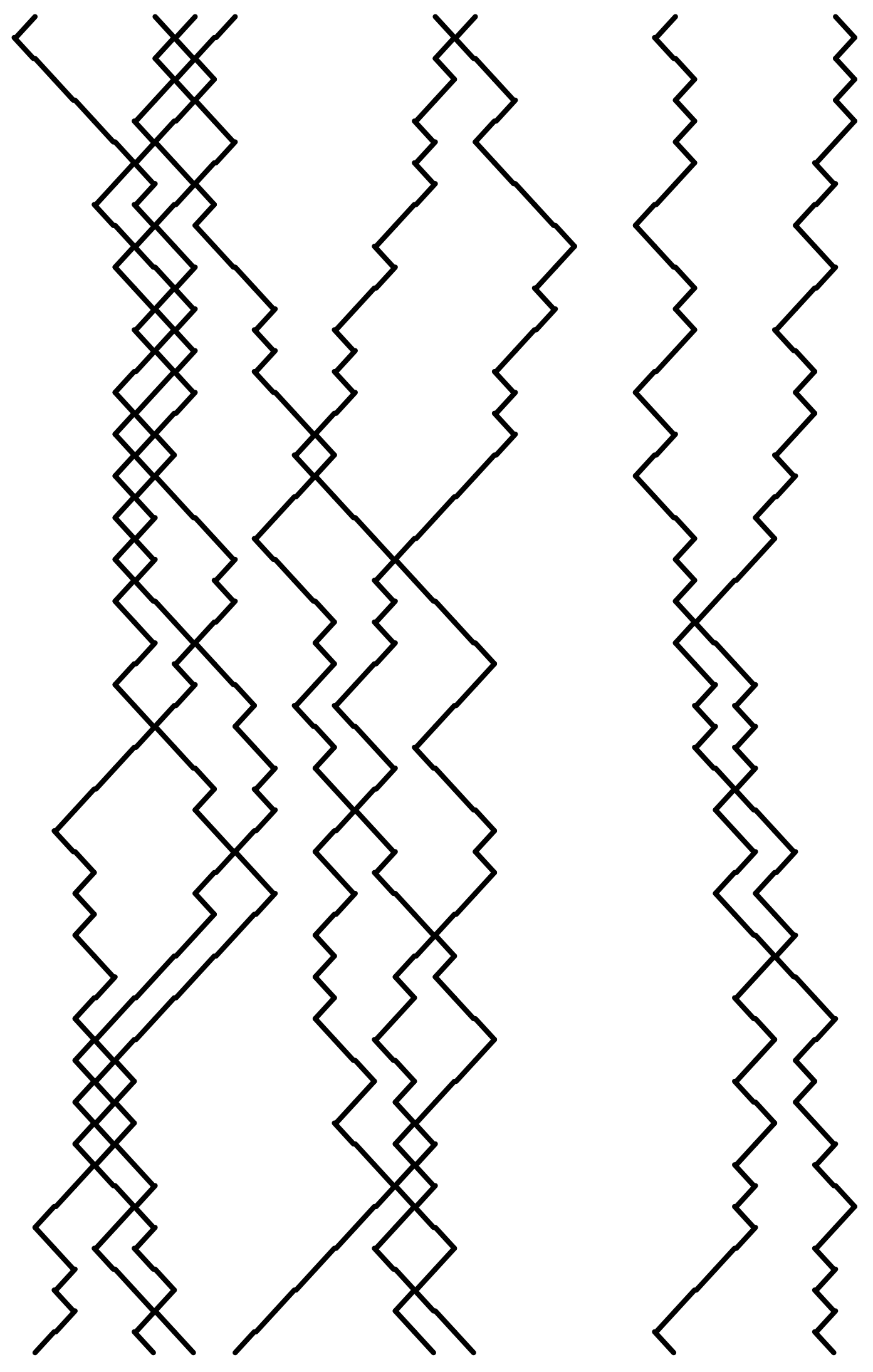



$\circ \bullet \bullet \bullet \circ q^{\circ} \bullet \rho \bullet$

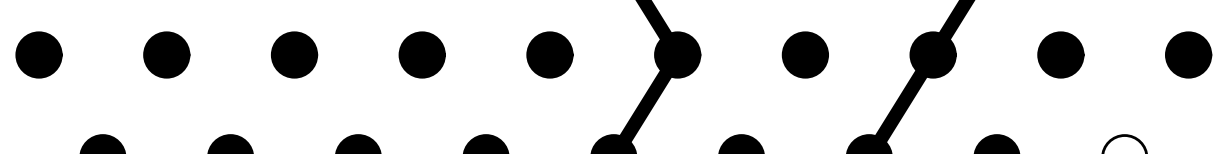
- $\bullet-\{0\{0$ - - 0 - 0$\} \circ\} \bullet$ - $\bullet-0$ - 0

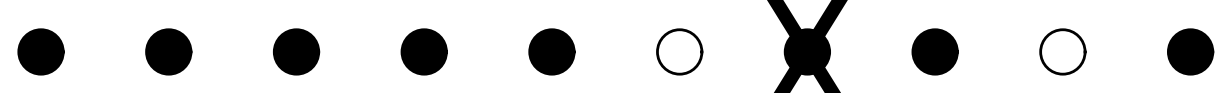
- - - 0 \& - - 0 o 0 - - 0 - 00$\}$ - 0 - 0 - 0 • $0 \bullet \bullet \bullet<\bullet$ - - 0 - 0 - - $0 \%$ - - 0 - 0 ० - $0 \bullet\{0\{0 \bullet \bullet$ - 0 - $\{0\}$ - - - 0$\} 0\} \bullet$ $\circ \bullet \bullet<0\} \bullet$ - - -0$\}$ - 0 - 


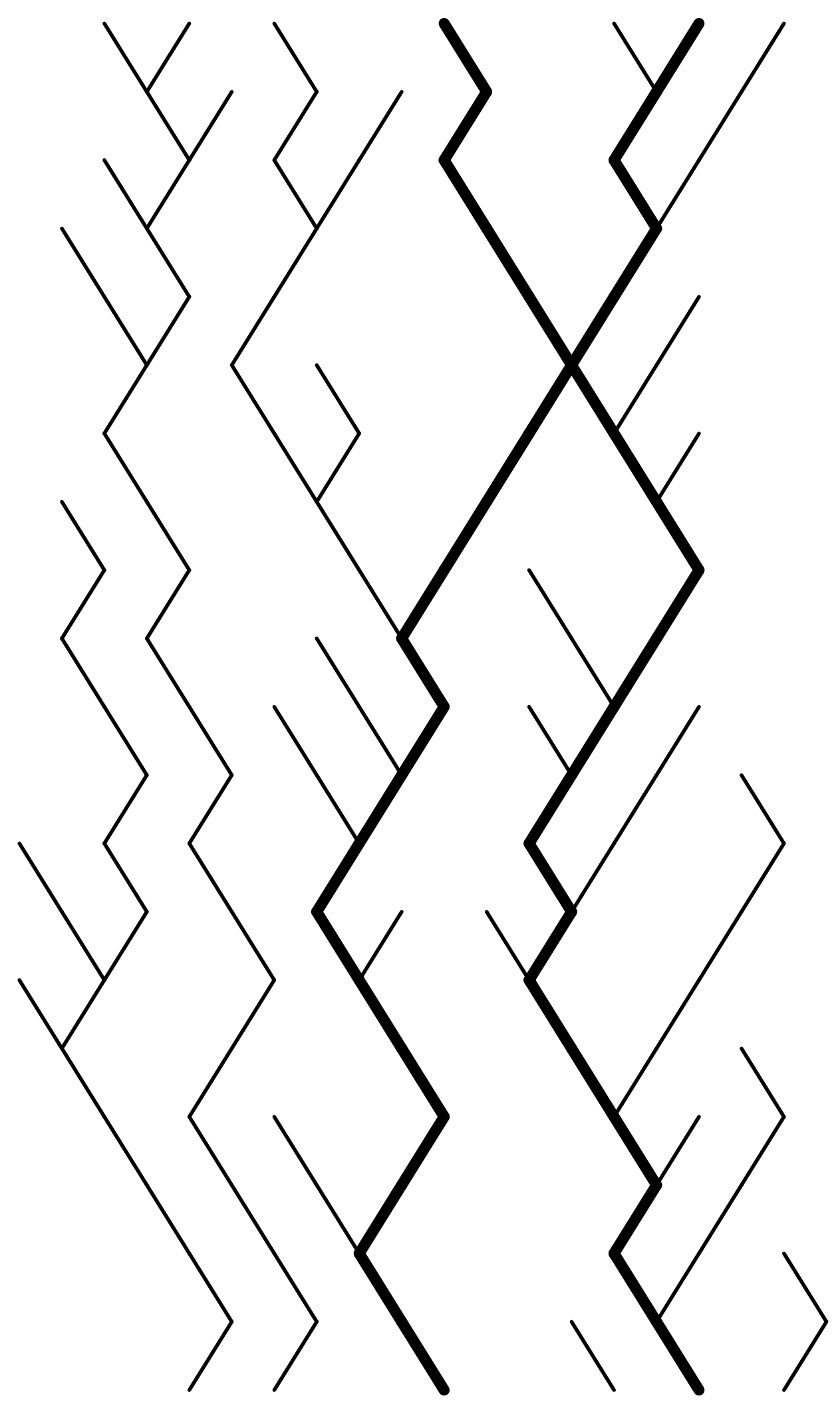

